\title{
Elliptic Hypergeometric Summations by Taylor Series Expansion and Interpolation ${ }^{\star}$
}

\author{
Michael J. SCHLOSSER and Meesue YOO \\ Fakultät für Mathematik, Universität Wien, \\ Oskar-Morgenstern-Platz 1, A-1090 Vienna, Austria \\ E-mail:michael.schlosser@univie.ac.at,meesue.yoo@univie.ac.at \\ URL: http://www.mat.univie.ac.at/ schlosse/
}

Received March 01, 2016, in final form April 13, 2016; Published online April 19, 2016

http://dx.doi.org/10.3842/SIGMA.2016.039

\begin{abstract}
We use elliptic Taylor series expansions and interpolation to deduce a number of summations for elliptic hypergeometric series. We extend to the well-poised elliptic case results that in the $q$-case have previously been obtained by Cooper and by Ismail and Stanton. We also provide identities involving S. Bhargava's cubic theta functions.
\end{abstract}

Key words: elliptic hypergeometric series; summations; Taylor series expansion; interpolation

2010 Mathematics Subject Classification: 30E05; 33D15; 33D70; 33E05; 33E20

\section{Introduction}

Previously, one of us [21] established an elliptic Taylor expansion theorem which extends Ismail's [11] expansion for functions symmetric in $z$ and $1 / z$ in terms of the Askey-Wilson monomial basis. The expansion theorem in [21] involves a special case of Rains' [17] elliptic extension of the Askey-Wilson divided difference operator. As applications, new simple proofs were given for Frenkel and Turaev's [8] elliptic extensions of Jackson's ${ }_{8} \phi_{7}$ summation and of Bailey's ${ }_{10} \phi_{9}$ transformation. A further application concerned the computation of the connection coefficients of Spiridonov's [23] elliptic extension of Rahman's biorthogonal rational functions.

Here we take a closer look at elliptic Taylor expansions. In particular, we describe the action of the $m$-th elliptic divided difference on a function, expressed in terms of the function. In the ordinary case, if $\delta_{h}$ denotes the central difference operator, defined by $\delta_{h} f(x)=f\left(x+\frac{h}{2}\right)-$ $f\left(x-\frac{h}{2}\right)$, the $m$-th difference is given by

$$
\delta_{h}^{m} f(x)=\sum_{k=0}^{m}(-1)^{k}\left(\begin{array}{c}
m \\
k
\end{array}\right) f\left(x+\left(\frac{m}{2}-k\right) h\right) .
$$

For the $q$-case, where $\delta_{h}$ is replaced by the Askey-Wilson operator $\mathcal{D}_{q}$, acting on functions $f(z)$ symmetric in $z$ and $1 / z$, an explicit formula for $\mathcal{D}_{q}^{m} f(z)$ was established by Cooper [5]. One of the results of our paper concerns an extension of Cooper's formula to the elliptic setting. We remark that Ismail, Rains and Stanton [12] independently have also proved an elliptic extension of Cooper's formula which turns out to be equivalent to our result by a multiplication of operators. In [14], Ismail and Stanton have used Cooper's explicit formula to work out an explicit interpolation formula for polynomials symmetric in $z$ and $1 / z$. Likewise, we use our elliptic extension of Cooper's formula to find an elliptic interpolation formula. Application of this formula yields single and multivariable identities of Karlsson-Minton type.

\footnotetext{
${ }^{\star}$ This paper is a contribution to the Special Issue on Orthogonal Polynomials, Special Functions and Applications. The full collection is available at http://www.emis.de/journals/SIGMA/OPSFA2015.html
} 
Ismail and Stanton [13] not only considered Taylor expansions in terms of the Askey-Wilson monomial basis $\left\{(a z, a / z ; q)_{n}, n \geq 0\right\}$ (see the subsequent subsection for the $q$-shifted factorial notation), but also in terms of the basis $\left\{\left(q^{\frac{1}{4}} z, q^{\frac{1}{4}} / z ; q^{\frac{1}{2}}\right)_{n}, n \geq 0\right\}$, for which they deduced quadratic summations as applications. We are able to extend Ismail and Stanton's analysis and provide, in particular, a Taylor expansion for an elliptic extension of this other basis. We note that in addition, Ismail and Stanton [13, Theorem 2.2] gave a Taylor expansion theorem for the basis $\left\{\left(1+z^{2}\right)\left(-q^{2-n} z^{2} ; q^{2}\right)_{n-1} z^{-n}, n \geq 0\right\}$, however this result (which involves an evaluation at $z=0)$ appears not to extend to the elliptic setting.

Finally, we consider series partially involving products of S. Bhargava's [3] cubic theta functions. Such series have not been considered before. We introduce two different cubic theta extensions of shifted factorials which are designed in such forms that they behave well under the iterated action of the elliptic Askey-Wilson operator. Applications of Taylor expansion yield cubic theta extensions of Jackson's ${ }_{8} \phi_{7}$ summation formula and of a quadratic summation of Gessel and Stanton.

Before we present our new results, to make this paper more self-contained, we briefly review some important material from the theory of elliptic hypergeometric series. Afterwards we turn to the Askey-Wilson operator and its elliptic extension, and then we provide our new results.

\section{$1.1 \quad$ Elliptic hypergeometric series}

For basic hypergeometric series, see Gasper and Rahman's textbook [9]. Elliptic hypergeometric series are treated there in Chapter 11.

By definition, a function is elliptic if it is meromorphic and doubly periodic. It is well known (cf., e.g., [25]) that elliptic functions can be built from quotients of theta functions.

As building blocks we will use the modified Jacobi theta function with argument $x$ and nome $p$, defined (in multiplicative notation) by

$$
\theta(x ; p)=\prod_{j \geq 0}\left(\left(1-p^{j} x\right)\left(1-p^{j+1} / x\right)\right), \quad \theta\left(x_{1}, \ldots, x_{m} ; p\right)=\prod_{k=1}^{m} \theta\left(x_{k} ; p\right),
$$

where $x, x_{1}, \ldots, x_{m} \neq 0,|p|<1$.

The modified Jacobi theta functions satisfy the following basic properties which are essential in the theory of elliptic hypergeometric series:

$$
\begin{aligned}
& \theta(x ; p)=-x \theta(1 / x ; p), \\
& \theta(p x ; p)=-\frac{1}{x} \theta(x ; p),
\end{aligned}
$$

and the addition formula

$$
\theta(x y, x / y, u v, u / v ; p)-\theta(x v, x / v, u y, u / y ; p)=\frac{u}{y} \theta(y v, y / v, x u, x / u ; p)
$$

(cf. [26, p. 451, Example 5]).

Note that in the theta function $\theta(x ; p)$ we cannot let $x \rightarrow 0$ (unless we first let $p \rightarrow 0$ ) for $x$ is a pole of infinite order.

Further, we define the theta shifted factorial (or $q, p$-shifted factorial) by

$$
(a ; q, p)_{n}= \begin{cases}\prod_{k=0}^{n-1} \theta\left(a q^{k} ; p\right), & n=1,2, \ldots, \\ 1, & n=0, \\ 1 / \prod_{k=0}^{-n-1} \theta\left(a q^{n+k} ; p\right), & n=-1,-2, \ldots,\end{cases}
$$


together with

$$
\left(a_{1}, a_{2}, \ldots, a_{m} ; q, p\right)_{n}=\prod_{k=1}^{m}\left(a_{k} ; q, p\right)_{n}
$$

for compact notation. For $p=0$ we have $\theta(x ; 0)=1-x$ and, hence, $(a ; q, 0)_{n}=(a ; q)_{n}$ is a $q$-shifted factorial in base $q$. The parameters $q$ and $p$ in $(a ; q, p)_{n}$ are called the base and nome, respectively. Observe that

$$
(p a ; q, p)_{n}=(-1)^{n} a^{-n} q^{-\left(\begin{array}{l}
n \\
2
\end{array}\right)}(a ; q, p)_{n},
$$

which follows from (1.1b). A list of other useful identities for manipulating the $q, p$-shifted factorials is given in [9, Section 11.2].

A series $\sum c_{n}$ is called an elliptic hypergeometric series if $g(n)=c_{n+1} / c_{n}$ is an elliptic function of $n$ with $n$ considered as a complex variable, i.e., the function $g(x)$ is a doubly periodic meromorphic function of the complex variable $x$. Without loss of generality, by the theory of theta functions, one may assume that

$$
g(x)=\frac{\theta\left(a_{1} q^{x}, a_{2} q^{x}, \ldots, a_{s+1} q^{x} ; p\right)}{\theta\left(q^{1+x}, b_{1} q^{x}, \ldots, b_{s} q^{x} ; p\right)} z,
$$

where the elliptic balancing condition, namely

$$
a_{1} a_{2} \cdots a_{s+1}=q b_{1} b_{2} \cdots b_{s},
$$

holds. If we write $q=e^{2 \pi i \sigma}, p=e^{2 \pi i \tau}$, with complex $\sigma, \tau$, then $g(x)$ is indeed periodic in $x$ with periods $\sigma^{-1}$ and $\tau \sigma^{-1}$.

For convergence reasons, one usually requires $a_{s+1}=q^{-n}$ ( $n$ being a nonnegative integer), so that the sum of an elliptic hypergeometric series is in fact finite.

Very-well-poised elliptic hypergeometric series are defined as

$$
{ }_{s+1} V_{s}\left(a_{1} ; a_{6}, \ldots, a_{s+1} ; q, p\right):=\sum_{k=0}^{\infty} \frac{\theta\left(a_{1} q^{2 k} ; p\right)}{\theta\left(a_{1} ; p\right)} \frac{\left(a_{1}, a_{6}, \ldots, a_{s+1} ; q, p\right)_{k}}{\left(q, a_{1} q / a_{6}, \ldots, a_{1} q / a_{s+1} ; q, p\right)_{k}}(q z)^{k},
$$

where

$$
q^{2} a_{6}^{2} a_{7}^{2} \cdots a_{s+1}^{2}=\left(a_{1} q\right)^{s-5} .
$$

Note that in the elliptic case the number of pairs of numerator and denominator parameters involved in the construction of the very-well-poised term $\theta\left(a_{1} q^{2 k} ; p\right) / \theta\left(a_{1} ; p\right)$ is four (whereas in the basic case this number is two, in the ordinary case only one). See Spiridonov [23] or Gasper and Rahman [9, Chapter 11] for details.

In their study of elliptic $6 j$ symbols (which are elliptic solutions of the Yang-Baxter equation found by Baxter [2] and Date et al. [7]), Frenkel and Turaev [8] discovered the following ${ }_{10} V_{9}$ summation formula (as a result of a more general ${ }_{12} V_{11}$ transformation, being a consequence of the tetrahedral symmetry of the elliptic $6 j$ symbols):

$$
{ }_{10} V_{9}\left(a ; b, c, d, e, q^{-n} ; q, p\right)=\frac{(a q, a q / b c, a q / b d, a q / c d ; q, p)_{n}}{(a q / b, a q / c, a q / d, a q / b c d ; q, p)_{n}},
$$

where $a^{2} q^{n+1}=b c d e$. The ${ }_{10} V_{9}$ summation is an elliptic analogue of Jackson's ${ }_{8} \phi_{7}$ summation formula (cf. [9, equation (2.6.2)])

$$
\sum_{k=0}^{n} \frac{\left(1-a q^{2 k}\right)\left(a, b, c, d, e, q^{-n} ; q\right)_{k}}{(1-a)\left(q, a q / b, a q / c, a q / d, a q / e, a q^{n+1} ; q\right)_{k}} q^{k}=\frac{(a q, a q / b c, a q / b d, a q / c d ; q)_{n}}{(a q / b, a q / c, a q / d, a q / b c d ; q)_{n}}
$$

where $a^{2} q^{n+1}=b c d e$, which in turn is a $q$-analogue of Dougall's ${ }_{7} F_{6}$ summation formula. 


\subsection{The Askey-Wilson operator}

The Askey-Wilson operator $\mathcal{D}_{q}$ was first defined in [1]. We consider meromorphic functions $f(z)$ symmetric in $z$ and $1 / z$. Writing $z=e^{i \theta}$ (note that $\theta$ need not to be real), we may consider $f$ to be a function in $x=\cos \theta=(z+1 / z) / 2$ and write $f[x]:=f(z)$. (I.e., $f$ can be considered as a function in $z$, or equivalently, as a function in $x$, where the two different notations specify the dependency to be considered.)

The Askey-Wilson operator acts on functions of $x=\cos \theta$. It is defined as follows:

$$
\mathcal{D}_{q} f[x]=\frac{f\left(q^{\frac{1}{2}} z\right)-f\left(q^{-\frac{1}{2}} z\right)}{\iota\left(q^{\frac{1}{2}} z\right)-\iota\left(q^{-\frac{1}{2}} z\right)},
$$

where $\iota[x]=x$ (i.e., $\iota(z)=(z+1 / z) / 2)$. Equation (1.4) can also be written as

$$
\mathcal{D}_{q} f[x]=\frac{f\left(q^{\frac{1}{2}} z\right)-f\left(q^{-\frac{1}{2}} z\right)}{i\left(q^{\frac{1}{2}}-q^{-\frac{1}{2}}\right) \sin \theta} .
$$

The operator $\mathcal{D}_{q}$ is a $q$-analogue of the differentiation operator (which is different to Jackson's $q$-difference operator). In particular, since

$$
\mathcal{D}_{q} T_{n}[x]=\frac{q^{\frac{n}{2}}-q^{-\frac{n}{2}}}{q^{\frac{1}{2}}-q^{-\frac{1}{2}}} U_{n-1}[x],
$$

where $T_{n}[\cos \theta]=\cos n \theta$ and $U_{n}[\cos \theta]=\sin (n+1) \theta / \sin \theta$ are the Chebyshev polynomials of the first and second kind, one easily sees that $\mathcal{D}_{q}$ maps polynomials to polynomials, lowering the degree by one.

In the calculus of the Askey-Wilson operator the so-called "Askey-Wilson monomials" $\phi_{n}(x ; a)=(a z, a / z ; q)_{n}$ form a natural basis for polynomials or power series in $x$. One readily computes

$$
\mathcal{D}_{q}(a z, a / z ; q)_{n}=-\frac{2 a\left(1-q^{n}\right)}{(1-q)}\left(a q^{\frac{1}{2}} z, a q^{\frac{1}{2}} / z ; q\right)_{n-1} .
$$

Ismail [11] proved the following Taylor theorem for polynomials $f[x]$.

Theorem 1.1. If $f[x]$ is a polynomial in $x$ of degree $n$, then

$$
f[x]=\sum_{k=0}^{n} f_{k} \phi_{k}(x ; a),
$$

where

$$
f_{k}=\frac{(q-1)^{k}}{(2 a)^{k}(q ; q)_{k}} q^{-k(k-1) / 4}\left[\mathcal{D}_{q}^{k} f[x]\right]_{x=x_{k}}, \quad x_{k}:=\frac{1}{2}\left(a q^{\frac{k}{2}}+q^{-\frac{k}{2}} / a\right) .
$$

As it was shown in [11], the application of Theorem 1.1 to $f(z)=(b z, b / z ; q)_{n}$ immediately gives the $q$-Pfaff-Saalschütz summation (cf. [9, equation (1.7.2)]), in the form

$$
\frac{(b z, b / z ; q)_{n}}{(b a, b / a ; q)_{n}}={ }_{3} \phi_{2}\left[\begin{array}{l}
a z, a / z, q^{-n} \\
a b, q^{1-n} a / b
\end{array} ; q, q\right],
$$

and its application to the Askey-Wilson polynomials,

$$
\omega_{n}(x ; a, b, c, d ; q):={ }_{4} \phi_{3}\left[\begin{array}{c}
a z, a / z, a b c d q^{n-1}, q^{-n} \\
a b, a c, a d
\end{array} ; q, q\right],
$$


gives a connection coefficient identity which, by specialization, can be reduced to the Sears transformation (cf. [9, equation (3.2.1)]), in the form

$$
\omega_{n}(x ; a, b, c, d ; q)=\frac{a^{n}(b c, b d ; q)_{n}}{b^{n}(a c, a d ; q)_{n}} \omega_{n}(x ; b, a, c, d ; q) .
$$

Ismail and Stanton [13] extended the above polynomial Taylor theorem to hold for entire functions of exponential growth, resulting in infinite Taylor expansions. Marco and Parcet [15] extended this yet further to hold for arbitrary $q$-differentiable functions, resulting in infinite Taylor expansions with explicit remainder term. Among other results they were able to recover the nonterminating $q$-Pfaff-Saalschütz summation (cf. [9, Appendix (II.24)]).

\subsection{The well-poised and elliptic Askey-Wilson operator}

Since

$$
\begin{gathered}
\mathcal{D}_{q} \frac{(a z, a / z ; q)_{n}}{(c z, c / z ; q)_{n}}=\frac{2}{\left(q^{\frac{1}{2}}-q^{-\frac{1}{2}}\right)(z-1 / z)}\left[\frac{\left(a q^{\frac{1}{2}} z, a q^{-\frac{1}{2}} / z ; q\right)_{n}}{\left(c q^{\frac{1}{2}} z, c q^{-\frac{1}{2}} / z ; q\right)_{n}}-\frac{\left(a q^{-\frac{1}{2}} z, a q^{\frac{1}{2}} / z ; q\right)_{n}}{\left(c q^{-\frac{1}{2}} z, c q^{\frac{1}{2}} / z ; q\right)_{n}}\right] \\
=\frac{2}{\left(q^{\frac{1}{2}}-q^{-\frac{1}{2}}\right)(z-1 / z)} \frac{\left(a q^{\frac{1}{2}} z, a q^{\frac{1}{2}} / z ; q\right)_{n-1}}{\left(c q^{\frac{1}{2}} z, c q^{\frac{1}{2}} / z ; q\right)_{n-1}} \\
\quad \times\left[\frac{\left(1-a z q^{n-\frac{1}{2}}\right)\left(1-a q^{-\frac{1}{2}} / z\right)}{\left(1-c z q^{n-\frac{1}{2}}\right)\left(1-c q^{-\frac{1}{2}} / z\right)}-\frac{\left(1-a z q^{-\frac{1}{2}}\right)\left(1-a q^{n-\frac{1}{2}} / z\right)}{\left(1-c z q^{-\frac{1}{2}}\right)\left(1-c q^{n-\frac{1}{2}} / z\right)}\right] \\
=\frac{(-1) 2 a(1-c / a)\left(1-a c q^{n-1}\right)\left(1-q^{n}\right)}{\left(1-c z q^{-\frac{1}{2}}\right)\left(1-c z q^{\frac{1}{2}}\right)\left(1-c q^{-\frac{1}{2}} / z\right)\left(1-c q^{\frac{1}{2}} / z\right)(1-q)} \frac{\left(a q^{\frac{1}{2}} z, a q^{\frac{1}{2}} / z ; q\right)_{n-1}}{\left(c q^{\frac{3}{2}} z, c q^{\frac{3}{2}} / z ; q\right)_{n-1}},
\end{gathered}
$$

we were led in [21] to define a $c$-generalized well-poised Askey-Wilson operator acting on $x$ (or $z$ ) by

$$
\mathcal{D}_{c, q}=\left(1-c z q^{-\frac{1}{2}}\right)\left(1-c z q^{\frac{1}{2}}\right)\left(1-c q^{-\frac{1}{2}} / z\right)\left(1-c q^{\frac{1}{2}} / z\right) \mathcal{D}_{q}
$$

which acts "degree-lowering" on the "rational monomials" (or "well-poised monomials")

$$
\frac{(a z, a / z ; q)_{n}}{(c z, c / z ; q)_{n}}
$$

in the form

$$
\mathcal{D}_{c, q} \frac{(a z, a / z ; q)_{n}}{(c z, c / z ; q)_{n}}=\frac{(-1) 2 a(1-c / a)\left(1-a c q^{n-1}\right)\left(1-q^{n}\right)}{(1-q)} \frac{\left(a q^{\frac{1}{2}} z, a q^{\frac{1}{2}} / z ; q\right)_{n-1}}{\left(c q^{\frac{3}{2}} z, c q^{\frac{3}{2}} / z ; q\right)_{n-1}} .
$$

Clearly, $\mathcal{D}_{0, q}=\mathcal{D}_{q}$

More generally, for parameters $c, q, p$ with $|q|,|p|<1$, we defined an elliptic extension of the Askey-Wilson operator, acting on functions symmetric in $z^{ \pm 1}$, by

$$
\mathcal{D}_{c, q, p} f(z)=2 q^{\frac{1}{2}} z \frac{\theta\left(c z q^{-\frac{1}{2}}, c z q^{\frac{1}{2}}, c q^{-\frac{1}{2}} / z, c q^{\frac{1}{2}} / z ; p\right)}{\theta\left(q, z^{2} ; p\right)}\left(f\left(q^{\frac{1}{2}} z\right)-f\left(q^{-\frac{1}{2}} z\right)\right) .
$$

Note that $\mathcal{D}_{c, q, 0}=\mathcal{D}_{c, q}$.

In particular, using (1.1c), we have

$$
\mathcal{D}_{c, q, p} \frac{(a z, a / z ; q, p)_{n}}{(c z, c / z ; q, p)_{n}}=\frac{(-1) 2 a \theta\left(c / a, a c q^{n-1}, q^{n} ; p\right)}{\theta(q ; p)} \frac{\left(a q^{\frac{1}{2}} z, a q^{\frac{1}{2}} / z ; q, p\right)_{n-1}}{\left(c q^{\frac{3}{2}} z, c q^{\frac{3}{2}} / z ; q, p\right)_{n-1}} .
$$


Remark 1.2. The operator $\mathcal{D}_{c, q, p}$ happens to be a special case of a multivariable difference operator introduced by Rains in [16]. Already in the single variable case Rains' operator involves two more parameters than $\mathcal{D}_{c, q, p}$. (Rains' difference operators generate a representation of the Sklyanin algebra, as observed in [16] and made explicit in [18] and [19, Section 6].) Rains' operator can be specialized to act as degree-lowering (as the above $\mathcal{D}_{c, q, p}$ does), degree-preserving or degree-raising on abelian functions. Rains used his multivariable difference operators in [16] to construct $B C_{n}$-symmetric biorthogonal abelian functions which generalize Koornwinder's orthogonal polynomials. He further used his operator in [17] to derive $B C_{n}$-symmetric extensions of Frenkel and Turaev's ${ }_{10} V_{9}$ summation and ${ }_{12} V_{11}$ transformation.

\section{Elliptic Taylor expansions and interpolation}

We work in the following space of abelian functions.

For a complex number $c$, let

$$
W_{c}^{n}:=\operatorname{span}_{\mathbb{C}}\left\{\frac{g_{k}(z)}{(c z, c / z ; q, p)_{k}}, 0 \leq k \leq n\right\},
$$

where $g_{k}(z)$ runs over all functions being holomorphic for $z \neq 0$ with $g_{k}(z)=g_{k}(1 / z)$ and

$$
g_{k}(p z)=\frac{1}{p^{k} z^{2 k}} g_{k}(z) .
$$

In classical terminology, $g_{k}(z)$ is an even theta function of order $2 k$ and zero characteristic. Rains [17] refers to such functions as $B C_{1}$ theta functions of degree $k$, whereas in Rosengren and Schlosser [20] they are referred to as $D_{k}$ theta functions. It is well-known that the space $V^{k}$ of even theta functions of order $2 k$ and zero characteristic has dimension $k+1$ (see, e.g., Weber [25, p. 49]).

Note that $W_{c}^{n}$ consists of certain abelian functions. (For $p \rightarrow 0$ these degenerate to certain rational functions which we may call "well-poised".)

Lemma 2.1 ([21, Lemma 4.1]). For any arbitrary but fixed complex number a (satisfying a $\neq$ $c q^{j} p^{k}$, for $j=0, \ldots, n-1$, and $k \in \mathbb{Z}$, and $a \neq q^{j} p^{k} / c$, for $j=2-2 n, \ldots, 1-n$, and $\left.k \in \mathbb{Z}\right)$, the set

$$
\left\{\frac{(a z, a / z ; q, p)_{k}}{(c z, c / z ; q, p)_{k}}, 0 \leq k \leq n\right\}
$$

forms a basis for $W_{c}^{n}$.

Note that, in view of (1.6), the elliptic Askey-Wilson operator maps functions in $W_{c}^{n}$ to functions in $W_{c q^{\frac{3}{2}}}^{n-1}$.

We now define

$$
\mathcal{D}_{c, q, p}^{(k)}=\underset{c q^{\frac{3}{2}, q, p}}{(k-1)} \mathcal{D}_{c, q, p},
$$

with $\mathcal{D}_{c, q, p}^{(0)}=\varepsilon$, the identity operator. We have the following elliptic Taylor expansion theorem which extends Theorem 1.1 of Ismail.

Theorem 2.2 ([21, Theorem 4.2]). If $f$ is in $W_{c}^{n}$, then

$$
f(z)=\sum_{k=0}^{n} f_{k} \frac{(a z, a / z ; q, p)_{k}}{(c z, c / z ; q, p)_{k}},
$$


where

$$
f_{k}=\frac{(-1)^{k} q^{-k(k-1) / 4} \theta(q ; p)^{k}}{(2 a)^{k}\left(q, c / a, a c q^{k-1} ; q, p\right)_{k}}\left[\mathcal{D}_{c, q, p}^{(k)} f(z)\right]_{z=a q^{\frac{k}{2}}} .
$$

Example 2.3. Let

$$
f(z)=\frac{(b z, b / z ; q, p)_{n}}{(c z, c / z ; q, p)_{n}}
$$

Application of Theorem 2.2 in conjunction with (1.6) gives

$$
\begin{aligned}
f_{k}= & \frac{(-1)^{k} q^{-k(k-1) / 4} \theta(q ; p)^{k}}{(2 a)^{k}\left(q, c / a, a c q^{k-1} ; q, p\right)_{k}} \\
& \times(-1)^{k}(2 b)^{k} q^{k(k-1) / 4} \frac{(q ; q, p)_{n}\left(c / b, b c q^{n-1} ; q, p\right)_{k}}{(q ; q, p)_{n-k} \theta(q ; p)^{k}} \frac{\left(a b q^{k}, b / a ; q, p\right)_{n-k}}{\left(a c q^{2 k}, c q^{k} / a ; q, p\right)_{n-k}} \\
= & \frac{(a b, b / a ; q, p)_{n}}{(a c, c / a ; q, p)_{n}} \frac{\theta\left(a c q^{2 k-1} ; p\right)}{\theta\left(a c q^{-1} ; p\right)} \frac{\left(a c q^{-1}, c / b, b c q^{n-1}, q^{-n} ; q, p\right)_{k}}{\left(q, a b, a q^{1-n} / b, a c q^{n} ; q, p\right)_{k}} q^{k},
\end{aligned}
$$

thus yielding Frenkel and Turaev's ${ }_{10} V_{9}$ summation (1.2), in the form

$$
\frac{(a c, c / a, b z, b / z ; q, p)_{n}}{(a b, b / a, c z, c / z ; q, p)_{n}}={ }_{10} V_{9}\left(a c q^{-1} ; a z, a / z, c / b, b c q^{n-1}, q^{-n} ; q, p\right) .
$$

We now prove an elliptic extension of a theorem of S. Cooper [5] which explicitly describes the action of the $m$-iterated Askey-Wilson operator.

Theorem 2.4. The action of $\mathcal{D}_{c, q, p}^{(m)}$ on a function $f \in W_{c}^{n}$ is given by

$$
\begin{aligned}
\mathcal{D}_{c, q, p}^{(m)} f(z)= & (-2 z)^{m} q^{\frac{m(3-m)}{4}} \frac{\left(c q^{\frac{m}{2}-1} z, c q^{\frac{m}{2}-1} / z ; q, p\right)_{m+1}}{(\theta(q ; p))^{m}} \\
& \times \sum_{k=0}^{m} q^{k(m-k)}\left[\begin{array}{c}
m \\
k
\end{array}\right]_{p, q} \frac{z^{2(k-m)}\left(c q^{\frac{m}{2}-k} z, c q^{-\frac{m}{2}+k} / z ; q, p\right)_{m-1}}{\left(q^{m-2 k+1} z^{2} ; q, p\right)_{k}\left(q^{2 k-m+1} z^{-2} ; q, p\right)_{m-k}} f\left(q^{\frac{m}{2}-k} z\right),
\end{aligned}
$$

where

$$
\left[\begin{array}{c}
m \\
k
\end{array}\right]_{p, q}=\frac{\left(q^{1+k} ; q, p\right)_{m-k}}{(q ; q, p)_{m-k}} .
$$

Proof. We prove this by induction. If $m=1$, then (2.1) just reduces to the definition of $\mathcal{D}_{c, q, p} f(z)$ in (1.5). Now say (2.1) holds up to some $m$. Then if we let $f^{(m)}(z):=\mathcal{D}_{c, q, p}^{(m)} f(z)$,

$$
\begin{aligned}
\mathcal{D}_{c, q, p}^{(m+1)} f(z)=\mathcal{D}_{c q^{\frac{3}{2} m}, q, p} f^{(m)}(z) \\
=2 q^{\frac{1}{2}} z \frac{\left(c z q^{\frac{3}{2} m-\frac{1}{2}}, c q^{\frac{3}{2} m-\frac{1}{2}} / z ; q, p\right)_{2}}{\theta\left(q, z^{2} ; p\right)}\left(f^{(m)}\left(q^{\frac{1}{2}} z\right)-f^{(m)}\left(q^{-\frac{1}{2}} z\right)\right) \\
=2 q^{\frac{1}{2}} z \frac{\left(c z q^{\frac{3}{2} m-\frac{1}{2}}, c q^{\frac{3}{2} m-\frac{1}{2}} / z ; q, p\right)_{2}}{\theta\left(q, z^{2} ; p\right)} \frac{(-2 z)^{m} q^{\frac{m(3-m)}{4}}}{(\theta(q ; p))^{m}}\left\{q^{\frac{m}{2}}\left(c q^{\frac{m}{2}-\frac{1}{2}} z, c q^{\frac{m}{2}-\frac{3}{2}} / z ; q, p\right)_{m+1}\right. \\
\quad \times \sum_{k=0}^{m} q^{(k-1)(m-k)}\left[\begin{array}{c}
m \\
k
\end{array}\right]_{p, q} \frac{z^{2(k-m)}\left(c q^{\frac{m}{2}-k+\frac{1}{2}} z, c q^{-\frac{m}{2}+k-\frac{1}{2}} / z ; q, p\right)_{m-1}}{\left(q^{m-2 k+2} z^{2} ; q, p\right)_{k}\left(q^{2 k-m} z^{-2} ; q, p\right)_{m-k}} f\left(q^{\frac{m}{2}-k+\frac{1}{2}} z\right)
\end{aligned}
$$




$$
\begin{aligned}
& -q^{-\frac{m}{2}}\left(c q^{\frac{m}{2}-\frac{3}{2}} z, c q^{\frac{m}{2}-\frac{1}{2}} / z ; q, p\right)_{m+1} \\
& \left.\times \sum_{k=0}^{m} q^{(k+1)(m-k)}\left[\begin{array}{c}
m \\
k
\end{array}\right]_{p, q} \frac{z^{2(k-m)}\left(c q^{\frac{m}{2}-k-\frac{1}{2}} z, c q^{-\frac{m}{2}+k+\frac{1}{2}} / z ; q, p\right)_{m-1}}{\left(q^{m-2 k} z^{2} ; q, p\right)_{k}\left(q^{2 k-m+2} z^{-2} ; q, p\right)_{m-k}} f\left(q^{\frac{m}{2}-k-\frac{1}{2}} z\right)\right\} \\
& =(2 z)^{m+1} q^{\frac{m(3-m)}{4}+\frac{m+1}{2}}(-1)^{m} \frac{\left(c z q^{\frac{3}{2} m-\frac{1}{2}}, c q^{\frac{3}{2} m-\frac{1}{2}} / z ; q, p\right)_{2}}{(\theta(q ; p))^{m+1} \theta\left(z^{2} ; p\right)}\left(c q^{\frac{m}{2}-\frac{1}{2}} z, c q^{\frac{m}{2}-\frac{1}{2}} / z ; q, p\right)_{m} \\
& \times\left\{\theta\left(c q^{\frac{3}{2} m-\frac{1}{2}} z, c q^{\frac{m}{2}-\frac{3}{2}} / z ; p\right)\right. \\
& \times \sum_{k=0}^{m} q^{(k-1)(m-k)}\left[\begin{array}{c}
m \\
k
\end{array}\right]_{p, q} \frac{z^{2(k-m)}\left(c q^{\frac{m}{2}-k+\frac{1}{2}} z, c q^{-\frac{m}{2}+k-\frac{1}{2}} / z ; q, p\right)_{m-1}}{\left(q^{m-2 k+2} z^{2} ; q, p\right)_{k}\left(q^{2 k-m} z^{-2} ; q, p\right)_{m-k}} f\left(q^{\frac{m}{2}-k+\frac{1}{2}} z\right) \\
& -q^{-m} \theta\left(c q^{\frac{m}{2}-\frac{3}{2}} z, c q^{\frac{3}{2} m-\frac{1}{2}} / z ; q, p\right) \\
& \left.\times \sum_{k=1}^{m+1} q^{k(m-k+1)}\left[\begin{array}{c}
m \\
k-1
\end{array}\right]_{p, q} \frac{z^{2(k-m-1)}\left(c q^{\frac{m}{2}-k+\frac{1}{2}} z, c q^{-\frac{m}{2}+k-\frac{1}{2}} / z ; q, p\right)_{m-1}}{\left(q^{m-2 k+2} z^{2} ; q, p\right)_{k-1}\left(q^{2 k-m} z^{-2} ; q, p\right)_{m-k+1}} f\left(q^{\frac{m}{2}-k+\frac{1}{2}} z\right)\right\} \\
& =(2 z)^{m+1} q^{\frac{m(3-m)}{4}+\frac{m+1}{2}}(-1)^{m} \frac{\left(c q^{\frac{m}{2}-\frac{1}{2}} z, c q^{\frac{m}{2}-\frac{1}{2}} / z ; q, p\right)_{m+2}}{(\theta(q ; p))^{m+1} \theta\left(z^{2} ; p\right)} \\
& \times\left\{\theta\left(c q^{\frac{3}{2} m-\frac{1}{2}} z, c q^{\frac{m}{2}-\frac{3}{2}} / z ; p\right) \times \frac{q^{-m} z^{-2 m}\left(c q^{\frac{m}{2}+\frac{1}{2}} z, c q^{-\frac{m}{2}-\frac{1}{2}} / z ; q, p\right)_{m-1}}{\left(q^{-m} z^{-2} ; q, p\right)_{n}} f\left(q^{\frac{m}{2}+\frac{1}{2}} z\right)\right. \\
& -q^{-m} \theta\left(c q^{\frac{m}{2}-\frac{3}{2}} z, c q^{\frac{3}{2} m-\frac{1}{2}} / z ; p\right) \times \frac{\left(c q^{-\frac{m}{2}-\frac{1}{2}} z, c q^{\frac{m}{2}+\frac{1}{2}} / z ; q, p\right)_{m-1}}{\left(q^{-m} z^{2} ; q, p\right)_{m}} f\left(q^{-\frac{m}{2}-\frac{1}{2}} z\right) \\
& +q^{-m} \sum_{k=1}^{m} q^{k(m-k+1)} \frac{\left(q^{1+k} ; q, p\right)_{m-k}}{(q ; q, p)_{m-k+1}} \frac{\left(c q^{\frac{m}{2}-k+\frac{1}{2}} z, c q^{-\frac{m}{2}+k-\frac{1}{2}} / z ; q, p\right)_{m-1} f\left(q^{\frac{m}{2}-k+\frac{1}{2}} z\right)}{\left(q^{m-2 k+2} z^{2} ; q, p\right)_{k}\left(q^{2 k-m} z^{-2} ; q, p\right)_{m-k+1}} \\
& \times z^{2 k-2 m}\left[\theta\left(q^{m-k+1}, c q^{\frac{3}{2} m-\frac{1}{2}} z, c q^{\frac{m}{2}-\frac{3}{2}} / z, q^{k} z^{-2} ; p\right)\right. \\
& \left.\left.-z^{-2} \theta\left(q^{k}, c q^{\frac{m}{2}-\frac{3}{2}} z, c q^{\frac{3}{2} m-\frac{1}{2}} / z, q^{m-k+1} z^{2} ; p\right)\right]\right\} \\
& =(-2 z)^{m+1} q^{\frac{(m+1)(2-m)}{4}} \frac{\left(c q^{\frac{m}{2}-\frac{1}{2}} z, c q^{\frac{m}{2}-\frac{1}{2}} / z ; q, p\right)_{m+2}}{(\theta(q ; p))^{m+1}} \\
& \times \sum_{k=0}^{m+1} q^{k(m+1-k)}\left[\begin{array}{c}
m+1 \\
k
\end{array}\right]_{p, q} \frac{z^{2(k-m-1)}\left(c q^{\frac{m+1}{2}-k} z, c q^{-\frac{m+1}{2}+k} / z ; q, p\right)_{m}}{\left(q^{m-2 k+2} z^{2} ; q, p\right)_{k}\left(q^{2 k-m} z^{-2} ; q, p\right)_{m+1-k}} f\left(q^{\frac{m+1}{2}-k} z\right) .
\end{aligned}
$$

Hence the theorem is proved. Notice that in the last step we used the addition formula (1.1c) with the substitutions

$$
(x, y, u, v) \mapsto\left(c q^{m-1}, q^{\frac{m+1}{2}} z, q^{\frac{m+1}{2}} / z, q^{\frac{m+1}{2}-k} z\right)
$$

to simplify the summand.

Remark 2.5. Our formula in Theorem 2.4 involves the $c$-dependent elliptic Askey-Wilson operator $\mathcal{D}_{c, q, p}^{(m)}$. Independently, Ismail, Rains and Stanton [12, Proposition 8.1] also gave an elliptic extension of Cooper's result. Although at first glance Ismail, Rains and Stanton's result, which involves a slightly different operator (without the denominator variable $c$ ), looks different from ours in Theorem 2.4, the two results are indeed equivalent up to a multiplication of operators. (We would like to thank Eric Rains for helping us to clarify this.) In fact, the operator

$$
\left(q^{\frac{m}{2}-1} c z, q^{\frac{m}{2}-1} c / z ; q, p\right)_{m+1}^{-1} \mathcal{D}_{c, q, p}^{(m)}(c z, c / z ; q, p)_{m-1}^{-1}
$$


(which acts on functions $h(z)=g(z) /\left(c z q^{m-1}, c q^{m-1} / z ; q, p\right)_{n-m+1}$ with $g(z)=g(1 / z)$ and $\left.g(p z)=p^{-n} z^{-2 n} g(z)\right)$ is independent of $c$, thus our operator $\mathcal{D}_{c, q, p}^{(m)}$ is proportional to

$$
v^{2 m} \theta\left(q^{\frac{m}{2}} v z, q^{\frac{m}{2}} v / z ; q\right)\left(q^{\frac{m}{2}-1} c z, q^{\frac{m}{2}-1} c / z ; q, p\right)_{m+1} \mathcal{D}_{m}(q ; p)(c z, c / z ; q, p)_{m-1} \theta(v z, v / z ; q)^{-1},
$$

with $\mathcal{D}_{m}(q ; p)$ from [12, Section 8], where the constant of proportionality is independent of $v$.

We are now able to obtain an elliptic extension of Ismail and Stanton's [14, Theorem 3.4] interpolation formula. In particular, for any $a \in \mathbb{C}$, the function $f \in W_{c}^{n}$ is uniquely determined by its evaluation at the $n+1$ interpolation points $a, a q, \ldots, a q^{n}$, with closed form coefficients.

Theorem 2.6. If $f$ is in $W_{c}^{n}$, then

$$
\begin{aligned}
& \frac{\left(a^{2} q, q, c z, c / z ; q, p\right)_{n}}{(a c, c / a, a q z, a q / z ; q, p)_{n}} f(z) \\
& \quad=\sum_{k=0}^{n} q^{k} \frac{\theta\left(a^{2} q^{2 k} ; p\right)}{\theta\left(a^{2} ; p\right)} \frac{\left(q^{-n}, a^{2}, a q / c, a c q^{n}, a z, a / z ; q, p\right)_{k}}{\left(q, a^{2} q^{n+1}, a c, a q^{1-n} / c, a q z, a q / z ; q, p\right)_{k}} f\left(a q^{k}\right) .
\end{aligned}
$$

Proof. By combining Theorem 2.2 and Theorem 2.4, we find that

$$
\begin{aligned}
f(z)= & \sum_{k=0}^{n} q^{k} \theta\left(c q^{-1} / a, a c q^{2 k-1} ; p\right) \frac{(a z, a / z ; q, p)_{k}}{(c z, c / z ; q, p)_{k}} \\
& \times \sum_{j=0}^{k} q^{-(k-j)^{2}} a^{2(j-k)} \frac{\left(a c q^{k-j}, c q^{-k+j} / a ; q, p\right)_{k-1}}{\left(q, a^{2} q^{2 k-2 j+1} ; q, p\right)_{j}\left(q, a^{-2} q^{2 j-2 k+1} ; q, p\right)_{k-j}} f\left(a q^{k-j}\right) .
\end{aligned}
$$

By shifting the index $k \mapsto k+j$, we get

$$
\begin{aligned}
f(z)= & \sum_{k=0}^{n} \sum_{j=0}^{n-k} q^{k+j} \theta\left(c q^{-1} / a, a c q^{2 k+2 j-1} ; p\right) \frac{(a z, a / z ; q, p)_{k+j}}{(c z, c / z ; q, p)_{k+j}} \\
& \times q^{-k^{2}} a^{-2 k} \frac{\left(a c q^{k}, c q^{-k} / a ; q, p\right)_{k+j-1}}{\left(q, a^{2} q^{2 k+1} ; q, p\right)_{j}\left(q, a^{-2} q^{-2 k+1} ; q, p\right)_{k}} f\left(a q^{k}\right) \\
= & \sum_{k=0}^{n} q^{k(1-k)} a^{-2 k} \frac{\left(a c q^{k}, c q^{-k} / a, a z, a / z ; q, p\right)_{k}}{\left(q, a^{-2} q^{-2 k+1}, c z, c / z ; q, p\right)_{k}} f\left(a q^{k}\right) \\
& \times \sum_{j=0}^{n-k} q^{j} \frac{\theta\left(a c q^{2 k+2 j-1} ; p\right)}{\theta\left(a c q^{2 k-1} ; p\right)} \frac{\left(a c q^{2 k-1}, c q^{-1} / a, a q^{k} z, a q^{k} / z, q^{-n+k}, a c q^{n+k} ; q, p\right)_{j}}{\left(q, a^{2} q^{2 k+1}, c q^{k} / z, c q^{k} z, q^{-n+k}, a c q^{n+k} ; q, p\right)_{j}} \\
= & \sum_{k=0}^{n} q^{k(1-k)} a^{-2 k} \\
& \times \frac{\left(a c q^{k}, c q^{-k} / a, a z, a / z ; q, p\right)_{k}\left(a c q^{2 k}, a q^{k+1} / z, a q^{k+1} z, c / a ; q, p\right)_{n-k}}{\left(q, a^{-2} q^{-2 k+1}, c z, c / z ; q, p\right)_{k}\left(q, a^{2} q^{2 k+1}, c q^{k} / z, c q^{k} z ; q, p\right)_{n-k}} f\left(a q^{k}\right) .
\end{aligned}
$$

The last sum was obtained by virtue of the Frenkel and Turaev summation formula (1.2). The theorem then follows by elementary manipulations.

Corollary 2.7. We have the elliptic Karlsson-Minton type identity

$$
\begin{aligned}
& \frac{\left(q, a^{2} q ; q, p\right)_{n}}{(a q z, a q / z ; q, p)_{n}} \frac{(b z, b / z ; q, p)_{s}(d z, d / z ; q, p)_{n-s}}{(a b, b / a ; q, p)_{s}(a d, d / a ; q, p)_{n-s}} \\
& \quad={ }_{12} V_{11}\left(a^{2}, q^{-n}, a z, a / z, a q / b, a q / d, a b q^{s}, a d q^{n-s} ; q, p\right) .
\end{aligned}
$$


Proof. We apply Theorem 2.6 to

$$
f(z)=\frac{(b z, b / z ; q, p)_{s}(d z, d / z ; q, p)_{n-s}}{(c z, c / z ; q, p)_{n}} .
$$

More generally, we have the following result.

Corollary 2.8. We have the elliptic Karlsson-Minton type identity

$$
\begin{aligned}
& \frac{\left(a^{2} q, q ; q, p\right)_{n}}{(a q z, a q / z ; q, p)_{n}} \prod_{j=1}^{n} \theta\left(b_{j} z, b_{j} / z ; p\right) \\
& \quad=\sum_{k=0}^{n} q^{k(n+1)} \frac{\theta\left(a^{2} q^{2 k} ; p\right)}{\theta\left(a^{2} ; p\right)} \frac{\left(q^{-n}, a^{2}, a z, a / z ; q, p\right)_{k}}{\left(q, a^{2} q^{n+1}, a q z, a q / z ; q, p\right)_{k}} \prod_{j=1}^{n} \theta\left(a b_{k} q^{k}, b_{j} q^{-k} / a ; p\right) .
\end{aligned}
$$

Proof. We take

$$
f(z)=\frac{\prod_{j=1}^{n} \theta\left(b_{j} z, b_{j} / z ; p\right)}{(c z, c / z ; q, p)_{n}}
$$

and apply Theorem 2.6.

Remark 2.9. It should be noted that if in the proof of Corollary 2.8 we instead would have taken

$$
f(z)=\frac{\prod_{j=1}^{t} \theta\left(b_{j} z, b_{j} / z ; p\right)}{(c z, c / z ; q, p)_{t}}
$$

for $0 \leq t \leq n$, we would have just obtained the special case of Corollary 2.8 with $b_{j} \mapsto c q^{j-1}$ for $t+1 \leq n$, which is clear carrying out those specializations in (2.2).

We now consider a multivariate version of Theorem 2.6. Let us consider the space of functions

$$
W_{c_{1}, \ldots, c_{m}}^{n_{1}, \ldots, n_{m}}:=\operatorname{Span}_{\mathbb{C}}\left\{\frac{g_{k_{1}, \ldots, k_{m}}\left(z_{1}, \ldots, z_{m}\right)}{\prod_{i=1}^{m}\left(c_{i} z_{i}, c_{i} / z_{i} ; q, p\right)_{k_{i}}}, 0 \leq k_{i} \leq n_{i}, i=1, \ldots, m\right\},
$$

where $g_{k_{1}, \ldots, k_{m}}\left(z_{1}, \ldots, z_{m}\right)$ runs over all functions being holomorphic in $z_{1}, z_{2}, \ldots, z_{m} \neq 0$ and symmetric in $z_{i}$ and $1 / z_{i}$, and

$$
g_{k_{1}, \ldots, k_{m}}\left(z_{1}, \ldots, p z_{i}, \ldots, z_{m}\right)=\frac{1}{p^{k_{i}} z_{i}^{2 k_{i}}} g_{k_{1}, \ldots, k_{m}}\left(z_{1}, \ldots, z_{i}, \ldots, z_{m}\right)
$$

for all $i=1, \ldots, m$.

We define a multivariate extension of the elliptic Askey-Wilson operator as follows.

$$
\begin{aligned}
\mathcal{D}_{c_{i}, q, p ; z_{i}} f\left(z_{1}, \ldots, z_{m}\right)= & 2 q^{\frac{1}{2}} z_{i} \frac{\theta\left(c_{i} z_{i} q^{-\frac{1}{2}}, c_{i} z_{i} q^{\frac{1}{2}}, c_{i} q^{-\frac{1}{2}} / z_{i}, c_{i} q^{\frac{1}{2}} / z_{i} ; p\right)}{\theta\left(q, z_{i}^{2} ; p\right)} \\
& \times\left(f\left(z_{1}, \ldots, q^{\frac{1}{2}} z_{i}, \ldots, z_{m}\right)-f\left(z_{1}, \ldots, q^{-\frac{1}{2}} z_{i}, \ldots, z_{m}\right)\right), \\
\mathcal{D}_{c_{i}, q, p ; z_{i}}^{(k+1)}=\mathcal{D}_{c_{i} q^{\frac{3}{2} k}, q, p ; z_{i}} \mathcal{D}_{c_{i}, q, p ; z_{i}}^{(k)}, &
\end{aligned}
$$

and for $\mathbf{c}=\left(c_{1}, \ldots, c_{m}\right), \mathbf{k}=\left(k_{1}, \ldots, k_{m}\right)$, and $\mathbf{z}=\left(z_{1}, \ldots, z_{m}\right)$,

$$
\mathcal{D}_{\mathbf{c}, q, p ; \mathbf{z}}^{(\mathbf{k})}=\mathcal{D}_{c_{1}, q, p ; z_{1}}^{\left(k_{1}\right)} \cdots \mathcal{D}_{c_{m}, q, p ; z_{m}}^{\left(k_{m}\right)} .
$$


Theorem 2.10. If $f\left(z_{1}, \ldots, z_{m}\right)$ is in $W_{\mathbf{c}}^{\mathbf{n}}$, then

$$
f\left(z_{1}, \ldots, z_{m}\right)=\sum_{k_{1}, \ldots, k_{m}=0}^{n_{1}, \ldots, n_{m}} f_{k_{1}, \ldots, k_{m}} \prod_{i=1}^{m} \frac{\left(a_{i} z_{i}, a_{i} / z_{i} ; q, p\right)_{k_{i}}}{\left(c_{i} z_{i}, c_{i} / z_{i} ; q, p\right)_{k_{i}}}
$$

where

$$
f_{k_{1}, \ldots, k_{m}}=\prod_{i=1}^{m} \frac{(-1)^{k_{i}} q^{-\frac{k_{i}\left(k_{i}-1\right)}{4}}(\theta(q ; p))^{k_{i}}}{\left(2 a_{i}\right)^{k_{i}}\left(q, c_{i} / a_{i}, a_{i} c_{i} q^{k_{i}-1} ; q, p\right)_{k_{i}}}\left[\mathcal{D}_{\mathbf{c}, q, p ; \mathbf{z}}^{(\mathbf{k})} f\left(z_{1}, \ldots, z_{m}\right)\right]_{z_{i}=a_{i} q^{k_{i} / 2}},
$$

where $\mathbf{k}=\left(k_{1}, \ldots, k_{m}\right)$.

Proof. Note that, for each $j=1, \ldots, m$,

$$
\begin{aligned}
& \mathcal{D}_{c_{j}, q, p ; z_{j}} \prod_{i=1}^{m} \frac{\left(a_{i} z_{i}, a_{i} / z_{i} ; q, p\right)_{n_{i}}}{\left(c_{i} z_{i}, c_{i} / z_{i} ; q, p\right)_{n_{i}}} \\
& =\frac{(-1) 2 a_{j} \theta\left(c_{j} / a_{j}, a_{j} c_{j} q^{n_{j}-1}, q^{n_{j}} ; p\right)}{\theta(q ; p)} \frac{\left(a_{j} q^{\frac{1}{2}} z_{j}, a_{j} q^{\frac{1}{2}} / z_{j} ; q, p\right)_{n_{j}-1}}{\left(c_{j} q^{\frac{3}{2}} z_{j}, c_{j} q^{\frac{3}{2}} / z_{j} ; q, p\right)_{n_{j}-1}} \prod_{\substack{i=1, i \neq j}}^{m} \frac{\left(a_{i} z_{i}, a_{i} / z_{i} ; q, p\right)_{n_{i}}}{\left(c_{i} z_{i}, c_{i} / z_{i} ; q, p\right)_{n_{i}}}
\end{aligned}
$$

Iterating (2.4) gives

$$
\begin{aligned}
{\left[\mathcal{D}_{\mathbf{c}, q, p ; \mathbf{z}}^{(\mathbf{k})} f\left(z_{1}, \ldots, z_{m}\right)\right]_{z_{i}=a_{i} q^{k_{i} / 2}} } \\
=\prod_{i=1}^{m}(-1)^{k_{i}}\left(2 a_{i}\right)^{k_{i}} q^{\frac{k_{i}\left(k_{i}-1\right)}{4}} \frac{(q ; q, p)_{n_{i}}\left(c_{i} / a_{i}, a_{i} c_{i} q^{n_{i}-1} ; q, p\right)_{k_{i}}}{(q ; q, p)_{n_{i}-k_{i}} \theta(q ; p)^{k_{i}}} \\
\quad \times\left[\frac{\left(a_{i} q^{\frac{k_{i}}{2}} z_{i}, a_{i} q^{\frac{k_{i}}{2}} / z_{i} ; q, p\right)_{n_{i}-k_{i}}}{\left(c_{i} q^{\frac{3 k_{i}}{2}} z_{i}, c_{i} q^{\frac{3 k_{i}}{2}} / z_{i} ; q, p\right)_{n_{i}-k_{i}}}\right]_{z_{i}=a_{i} q^{\frac{k_{i}}{2}}} \\
=\prod_{i=1}^{m}(-1)^{k_{i}}\left(2 a_{i}\right)^{k_{i}} q^{\frac{k_{i}\left(k_{i}-1\right)}{4}} \frac{\left(q, c_{i} / a_{i}, a_{i} c_{i} q^{k_{i}-1} ; q, p\right)_{k_{i}}}{\theta(q ; p)^{k_{i}}} \delta_{n_{i} k_{i} .} .
\end{aligned}
$$

Then the theorem follows by applying $\mathcal{D}_{\mathbf{c}, q, p ; \mathbf{z}}^{(\mathbf{j})}$ to both sides of $(2.3)$ and then setting $z_{i}=a_{i} q^{j_{i} / 2}$, for $i=1, \ldots, m$ and $\mathbf{j}=\left(j_{1}, \ldots, j_{m}\right)$.

Now we provide a multivariate extension of Theorem 2.4.

Theorem 2.11. For $\mathbf{n}=\left(n_{1}, \ldots, n_{m}\right)$, $\mathbf{c}=\left(c_{1}, \ldots, c_{m}\right)$,

$$
\begin{aligned}
& \mathcal{D}_{\mathbf{c}, q, p ; \mathbf{z}}^{(\mathbf{n})} f\left(z_{1}, \ldots, z_{m}\right)=\prod_{i=1}^{m}\left[\left(-2 z_{i}\right)^{n_{i}} q^{\frac{n_{i}\left(3-n_{i}\right)}{4}} \frac{\left(c_{i} q^{\frac{n_{i}}{2}-1} z_{i}, c_{i} q^{\frac{n_{i}}{2}-1} / z_{i} ; q, p\right)_{n_{i}+1}}{(\theta(q ; p))^{n_{i}}}\right] \\
& \times \sum_{k_{m}=0}^{n_{m}} \cdots \sum_{k_{1}=0}^{n_{1}} \prod_{i=1}^{m}\left(q^{k_{i}\left(n_{i}-k_{i}\right)}\left[\begin{array}{c}
n_{i} \\
k_{i}
\end{array}\right]_{p, q} \frac{z_{i}^{2\left(k_{i}-n_{i}\right)}\left(c_{i} q^{\frac{n_{i}}{2}-k_{i}} z_{i}, c_{i} q^{-\frac{n_{i}}{2}+k_{i}} / z_{i} ; q, p\right)_{n_{i}-1}}{\left(q^{n_{i}-2 k_{i}+1} z_{i}^{2} ; q, p\right)_{k_{i}}\left(q^{2 k_{i}-n_{i}+1} z_{i}^{-2} ; q, p\right)_{n_{i}-k_{i}}}\right. \\
&\left.\quad \times f\left(q^{\frac{n_{1}}{2}-k_{1}} z_{1}, \ldots, q^{\frac{n_{m}}{2}-k_{m}} z_{m}\right)\right) .
\end{aligned}
$$

Proof. The theorem follows by applying Theorem 2.4 successively for each $i=1, \ldots, m$. 
We combine Theorems 2.10 and 2.11 to obtain the following multivariable elliptic interpolation formula.

Theorem 2.12. For $f\left(z_{1}, \ldots, z_{m}\right)$ in $W_{\mathbf{c}}^{\mathbf{n}}$, we have

$$
\begin{aligned}
& \prod_{i=1}^{m} \frac{\left(a_{i}^{2} q, q, c_{i} z_{i}, c_{i} / z_{i} ; q, p\right)_{n_{i}}}{\left(a_{i} c_{i}, c_{i} / a_{i}, a_{i} q z_{i}, a_{i} q / z_{i} ; q, p\right)_{n_{i}}} f\left(z_{1}, \ldots, z_{m}\right) \\
& \quad \sum^{n_{1}, \ldots, n_{m}} \prod_{\substack{k_{1}, \ldots, k_{m}=0 \\
n}}^{m} q^{k_{i}} \frac{\theta\left(a_{i}^{2} q^{2 k_{i}} ; p\right)}{\theta\left(a_{i}^{2} ; p\right)} \frac{\left(q^{-n_{i}}, a_{i}^{2}, a_{i} q / c_{i}, a_{i} c_{i} q^{n_{i}}, a_{i} z_{i}, a_{i} / z_{i} ; q, p\right)_{k_{i}}}{\left(q, a_{i}^{2} q^{n_{i}+1}, a_{i} c_{i}, a_{i} q^{1-n_{i}} / c_{i}, a_{i} q z_{i}, a_{i} q / z_{i} ; q, p\right)_{k_{i}}} \\
& \quad \times f\left(a_{1} q^{k_{1}}, \ldots, a_{m} q^{k_{m}}\right) .
\end{aligned}
$$

This theorem extends a result given by Ismail and Stanton [14, Theorem 3.10], which can be obtained by taking $m=2, p \rightarrow 0, c_{1}=c_{2}=0$ and $n_{1}=n_{2}=n$.

Corollary 2.13. We have the following multivariable elliptic Karlsson-Minton type identity

$$
\begin{aligned}
& \prod_{i=1}^{m}\left(\frac{\left(a_{i}^{2} q, q ; q, p\right)_{n_{i}}}{\left(a_{i} q z_{i}, a_{i} q / z_{i} ; q, p\right)_{n_{i}}} \prod_{j=1}^{s_{i}} \frac{\left(b_{i j} z_{i}, b_{i j} / z_{i} ; q, p\right)_{v_{i j}}}{\left(b_{i j} a_{i}, b_{i j} / a_{i} ; q, p\right)_{v_{i j}}}\right) \prod_{1 \leq i<j \leq m}\left(a_{i}^{w_{i j}} z_{i}^{-w_{i j}} \theta\left(z_{i} z_{j}, z_{i} / z_{j} ; p\right)^{w_{i j}}\right. \\
& \left.\times \prod_{l_{i j}=1}^{r_{i j}} \frac{\left(\alpha_{l_{i j}} z_{i} z_{j}, \alpha_{l_{i j}} z_{i} / z_{j}, \alpha_{l_{i j}} z_{j} / z_{i}, \alpha_{l_{i j}} / z_{i} z_{j} ; q, p\right)_{u_{l i j}}}{\left(\alpha_{l_{i j}} a_{i} a_{j}, \alpha_{l_{i j}} a_{i} / a_{j}, \alpha_{l_{i j}} a_{j} / a_{i}, \alpha_{l_{i j}} / a_{i} a_{j} ; q, p\right)_{u_{l i j}}}\right) \\
& =\sum_{k_{1}, \ldots, k_{m}=0}^{n_{1}, \ldots, n_{m}} \prod_{i=1}^{m}\left(q^{k_{i}} \frac{\theta\left(a_{i}^{2} q^{2 k_{i}} ; p\right)}{\theta\left(a_{i}^{2} ; p\right)} \frac{\left(q^{-n_{i}}, a_{i}^{2}, a_{i} z_{i}, a_{i} / z_{i} ; q, p\right)_{k_{i}}}{\left(q, a_{i}^{2} q^{n_{i}+1}, a_{i} q z_{i}, a_{i} q / z_{i} ; q, p\right)_{k_{i}}}\right. \\
& \left.\times \prod_{j=1}^{s_{i}} \frac{\left(a_{i} b_{i j} q^{v_{i j}}, a_{i} q / b_{i j} ; q, p\right)_{k_{i}}}{\left(a_{i} b_{i j}, a_{i} q^{1-v_{i j}} / b_{i j} ; q, p\right)_{k_{i}}}\right) \\
& \times \prod_{1 \leq i<j \leq m} \prod_{l_{i j}=1}^{r_{i j}} q^{-2 u_{l_{i j}} k_{i}} \\
& \times \frac{\left(\alpha_{l_{i j}} a_{i} a_{j} q^{u_{l_{i j}}}, q a_{i} a_{j} / \alpha_{l_{i j}} ; q, p\right)_{k_{i}+k_{j}}\left(\alpha_{l_{i j}} a_{i} q^{u_{l_{i j}}} / a_{j}, q a_{i} / a_{j} \alpha_{l_{i j}} ; q, p\right)_{k_{i}-k_{j}}}{\left(\alpha_{l_{i j}} a_{i} a_{j}, q^{1-u_{l_{i j}}} a_{i} a_{j} / \alpha_{l_{i j}} ; q, p\right)_{k_{i}+k_{j}}\left(\alpha_{l_{i j}} a_{i} / a_{j}, q^{1-u_{l_{i j}}} a_{i} / a_{j} \alpha_{l_{i j}} ; q, p\right)_{k_{i}-k_{j}}} \\
& \times \prod_{1 \leq i<j \leq m} q^{-w_{i j} k_{i}} \theta\left(a_{i} a_{j} q^{k_{i}+k_{j}}, a_{i} q^{k_{i}-k_{j}} / a_{j} ; p\right)^{w_{i j}},
\end{aligned}
$$

where

$$
n_{i}=\sum_{j=1}^{s_{i}} v_{i j}+\sum_{j=1}^{i-1} w_{j i}+\sum_{j=i+1}^{m} w_{i j}+2 \sum_{l=1}^{r_{i j}}\left(\sum_{j=1}^{i-1} u_{l_{j i}}+\sum_{j=i+1}^{m} u_{l_{i j}}\right),
$$

for $i=1, \ldots, m$.

Proof. We apply Theorem 2.12 to

$$
\begin{aligned}
f\left(z_{1}, \ldots, z_{m}\right)= & \prod_{i=1}^{m} \frac{\prod_{j=1}^{s_{i}}\left(b_{i j} z_{i}, b_{i j} / z_{i} ; q, p\right)_{v_{i j}}}{\left(c_{i} z_{i}, c_{i} / z_{i} ; q, p\right)_{n_{i}}} \prod_{1 \leq i<j \leq m} z_{i}^{-w_{i j}} \theta\left(z_{i} z_{j}, z_{i} / z_{j} ; p\right)^{w_{i j}} \\
& \times \prod_{1 \leq i<j \leq m} \prod_{l_{i j}=1}^{r_{i j}}\left(\alpha_{l_{i j}} z_{i} z_{j}, \alpha_{l_{i j}} z_{i} / z_{j}, \alpha_{l_{i j}} z_{j} / z_{i}, \alpha_{l_{i j}} / z_{i} z_{j} ; q, p\right)_{u_{l_{i j}}},
\end{aligned}
$$


where

$$
n_{i}=\sum_{j=1}^{s_{i}} v_{i j}+\sum_{j=1}^{i-1} w_{j i}+\sum_{j=i+1}^{m} w_{i j}+2 \sum_{j=1}^{i-1} \sum_{l_{j i}=1}^{r_{j i}} u_{l_{j i}}+2 \sum_{j=i+1}^{m} \sum_{l_{i j}=1}^{r_{i j}} u_{l_{i j}},
$$

for $i=1, \ldots, m$.

Corollary 2.13 extends a result by Ismail and Stanton (see [14, Corollary 3.11]), corresponding to a special case of its $m=2$ instance.

More generally, $f\left(z_{1}, \ldots, z_{m}\right)$ could involve symmetrized products of $2^{k}$ factors of the form $\left(\lambda z_{i_{1}}^{ \pm} z_{i_{2}}^{ \pm} \cdots z_{i_{k}}^{ \pm} ; q, p\right)_{y}$ (the notation $z_{i_{j}}^{ \pm}$means that the resprective variable could appear as $z_{i_{j}}$ or $z_{i_{j}}^{-1}$, where all possible combinations appear), where $\left\{i_{1}, \ldots, i_{k}\right\}$ is any subset of $\{1, \ldots, n\}$. (In the corollary, we only considered factors for $k=1,2$.)

Corollary 2.13 can be easily seen to be equivalent to its $u_{l_{i j}}=1$ and $v_{i j}=1$ case, for all $i, j$, in which case the respective factorials reduce to simple theta functions. To recover the general case from this special case one can suitably increase $r_{i j}$ and $s_{1}, \ldots, s_{m}$ and choose the parameters partially in geometric progression to obtain shifted factorials. In particular, we can replace $r_{i j}$ by $u_{1}+\cdots+u_{r_{i j}}$ and relabel $\alpha_{u_{1}+\cdots+u_{l_{i j}-1}+h} \mapsto \alpha_{l_{i j}} q^{h-1}$, for all $1 \leq l_{i j} \leq r_{i j}, 1 \leq h \leq u_{l_{i j}}$, etc. (One could even add extra bases, in addition to $q$. This feature is typical for series of Karlsson-Minton type.)

For convenience, we restate the corollary in this equivalent form.

Corollary 2.14. We have the following multivariable elliptic Karlsson-Minton type identity

$$
\begin{aligned}
& \prod_{i=1}^{m}\left(\frac{\left(a_{i}^{2} q, q ; q, p\right)_{n_{i}}}{\left(a_{i} q z_{i}, a_{i} q / z_{i} ; q, p\right)_{n_{i}}} \prod_{j=1}^{s_{i}} \frac{\theta\left(b_{i j} z_{i}, b_{i j} / z_{i} ; p\right)}{\theta\left(b_{i j} a_{i}, b_{i j} / a_{i} ; p\right)}\right) \\
& \times \prod_{1 \leq i<j \leq m}\left(a_{i}^{w_{i j}} z_{i}^{-w_{i j}} \theta\left(z_{i} z_{j}, z_{i} / z_{j} ; p\right)^{w_{i j}} \prod_{l_{i j}=1}^{r_{i j}} \frac{\theta\left(\alpha_{l_{i j}} z_{i} z_{j}, \alpha_{l_{i j}} z_{i} / z_{j}, \alpha_{l_{i j}} z_{j} / z_{i}, \alpha_{l_{i j}} / z_{i} z_{j} ; p\right)}{\theta\left(\alpha_{l_{i j}} a_{i} a_{j}, \alpha_{l_{i j}} a_{i} / a_{j}, \alpha_{l_{i j}} a_{j} / a_{i}, \alpha_{l_{i j}} / a_{i} a_{j} ; p\right)}\right) \\
& =\sum_{k_{1}, \ldots, k_{m}=0}^{n_{1}, \ldots, n_{m}} \prod_{i=1}^{m}\left(q^{k_{i}} \frac{\theta\left(a_{i}^{2} q^{2 k_{i}} ; p\right)}{\theta\left(a_{i}^{2} ; p\right)} \frac{\left(q^{-n_{i}}, a_{i}^{2}, a_{i} z_{i}, a_{i} / z_{i} ; q, p\right)_{k_{i}}}{\left(q, a_{i}^{2} q^{n_{i}+1}, a_{i} q z_{i}, a_{i} q / z_{i} ; q, p\right)_{k_{i}}} \prod_{j=1}^{s_{i}} \frac{\theta\left(a_{i} b_{i j} q^{k_{i}}, a_{i} q^{k_{i}} / b_{i j} ; p\right)}{\theta\left(a_{i} b_{i j}, a_{i} / b_{i j} ; p\right)}\right) \\
& \times \prod_{1 \leq i<j \leq m} \prod_{l_{i j}=1}^{r_{i j}} q^{-2 k_{i}} \frac{\theta\left(\alpha_{l_{i j}} a_{i} a_{j} q^{k_{i}+k_{j}}, q^{k_{i}+k_{j}} a_{i} a_{j} / \alpha_{l_{i j}}, \alpha_{l_{i j}} a_{i} q^{k_{i}-k_{j}} / a_{j}, q^{k_{i}-k_{j}} a_{i} / a_{j} \alpha_{l_{i j}} ; p\right)}{\theta\left(\alpha_{l_{i j}} a_{i} a_{j}, a_{i} a_{j} / \alpha_{l_{i j}}, \alpha_{l_{i j}} a_{i} / a_{j}, a_{i} / a_{j} \alpha_{l_{i j}} ; p\right)} \\
& \times \prod_{1 \leq i<j \leq m} q^{-w_{i j} k_{i}} \theta\left(a_{i} a_{j} q^{k_{i}+k_{j}}, a_{i} q^{k_{i}-k_{j}} / a_{j} ; p\right)^{w_{i j}},
\end{aligned}
$$

where

$$
n_{i}=s_{i}+\sum_{j=1}^{i-1} w_{j i}+\sum_{j=i+1}^{m} w_{i j}+2 \sum_{j=1}^{i-1} r_{j i}+2 \sum_{j=i+1}^{m} r_{i j},
$$

for $i=1, \ldots, m$.

\subsection{A quadratic elliptic Taylor expansion theorem}

In [13] Ismail and Stanton also considered the basis $\left\{\phi_{k}(z), 0 \leq k \leq n\right\}$ where $\phi_{k}(z)=$ $\left(q^{1 / 4} z, q^{1 / 4} / z ; q^{1 / 2}\right)_{k}$. The set

$$
\left\{\frac{\left(q^{1 / 4} z, q^{1 / 4} / z ; q^{1 / 2}, p\right)_{k}}{(c z, c / z ; q, p)_{k}}, 0 \leq k \leq n\right\}
$$


apparently forms a basis for $W_{c}^{n}$. We now provide a Taylor expansion theorem with respect to this basis.

Theorem 2.15. If $f$ is in $W_{c}^{n}$, then

$$
f(z)=\sum_{k=0}^{n} f_{k} \frac{\left(q^{1 / 4} z, q^{1 / 4} / z ; q^{1 / 2}, p\right)_{k}}{(c z, c / z ; q, p)_{k}}
$$

where

$$
f_{k}=\frac{(-1)^{k} q^{-k / 4} \theta(q ; p)^{k}}{2^{k}(q ; q, p)_{k}\left(c q^{\frac{k}{2}-\frac{3}{4}} ; q^{1 / 2}, p\right)_{2 k}}\left[\mathcal{D}_{c, q, p}^{(k)} f(z)\right]_{z=q^{1 / 4}} .
$$

Proof. Note that

$$
\begin{aligned}
\mathcal{D}_{c, q, p}^{(k)} & \left(\frac{\left(q^{1 / 4} z, q^{1 / 4} / z ; q^{1 / 2}, p\right)_{n}}{(c z, c / z ; q, p)_{n}}\right) \\
\quad= & \frac{(-2)^{k} q^{k / 4}\left(c q^{\frac{n}{2}-\frac{3}{4}} ; q^{1 / 2}, p\right)_{2 k}(q ; q, p)_{n}}{(q ; q, p)_{n-k} \theta(q ; p)^{k}} \frac{\left(q^{1 / 4} z, q^{1 / 4} / z ; q^{1 / 2}, p\right)_{n-k}}{\left(c q^{\frac{3}{2} k} z, c q^{\frac{3}{2} k} / z ; q, p\right)_{n-k}},
\end{aligned}
$$

which can be proved by induction. The theorem then follows by applying $\mathcal{D}_{c, q, p}^{(j)}$ to both sides of (2.5) and then setting $z=q^{1 / 4}$.

In the following, we recover an elliptic quadratic summation by Warnaar [24, Corollary 4.4; $b=a]$, which was originally proved by using inverse relations. Its $p=0$ case has been given earlier by Gessel and Stanton [10, equation (1.4)].

Corollary 2.16. We have the following summation

$$
\begin{aligned}
& \frac{(a z, a / z ; q, p)_{n}}{(c z, c / z ; q, p)_{n}} \frac{\left(c q^{-1 / 4} ; q^{1 / 2}, p\right)_{2 n}}{\left(a q^{-1 / 4} ; q^{1 / 2}, p\right)_{2 n}} \\
& \quad=\sum_{k=0}^{n} q^{\frac{k}{2}} \frac{\theta\left(c q^{\frac{3}{2} k-\frac{3}{4}} ; p\right)}{\theta\left(c q^{-\frac{3}{4}} ; p\right)} \frac{\left(c / a, a c q^{n-1}, q^{-n} ; q, p\right)_{k}}{(c z, c / z, q ; q, p)_{k}} \frac{\left(c q^{-\frac{3}{4}}, q^{\frac{1}{4}} z, q^{\frac{1}{4}} / z ; q^{1 / 2}, p\right)_{k}}{\left(a q^{-\frac{1}{4}}, c q^{n-\frac{1}{4}}, q^{\frac{3}{4}-n} / a ; q^{1 / 2}, p\right)_{k}} .
\end{aligned}
$$

Proof. We apply Theorem 2.15 to

$$
f(z)=\frac{(a z, a / z ; q, p)_{n}}{(c z, c / z ; q, p)_{n}}
$$

Remark 2.17. If we expand

$$
\frac{\left(q^{\frac{1}{4}} z, q^{\frac{1}{4}} / z ; q^{\frac{1}{2}}, p\right)_{n}}{(c z, c / z ; q, p)_{n}}
$$

in terms of

$$
\frac{(a z, a / z ; q, p)_{n}}{(c z, c / z ; q, p)_{n}}
$$

using Theorem 2.2, we obtain

$$
\frac{\left(q^{\frac{1}{4}} z, q^{\frac{1}{4}} / z ; q^{\frac{1}{2}}, p\right)_{n}}{\left(a q^{\frac{1}{4}}, q^{\frac{1}{4}} / a ; q^{\frac{1}{2}}, p\right)_{n}} \frac{(a c, c / a ; q, p)_{n}}{(c z, c / z ; q, p)_{n}}
$$




$$
=\sum_{k=0}^{n} q^{k} \frac{\theta\left(a c q^{2 k-1} ; p\right)}{\theta\left(a c q^{-1} ; p\right)} \frac{\left(q^{-n}, a c q^{-1}, a z, a / z, c q^{\frac{n}{2}-\frac{3}{4}}, c q^{\frac{n}{2}-\frac{1}{4}} ; q, p\right)_{n}}{\left(q, a c q^{n}, c z, c / z, a q^{\frac{1}{4}-\frac{n}{2}}, a q^{\frac{3}{4}-\frac{n}{2}} ; q, p\right)_{n}} .
$$

At first glance this appears to be a true quadratic summation formula. However, the right-hand side of $(2.6)$ is

$$
{ }_{10} V_{9}\left(a c q^{-1} ; a z, a / z, c q^{\frac{n}{2}-\frac{3}{4}}, c q^{\frac{n}{2}-\frac{1}{4}}, q^{-n} ; q, p\right),
$$

which, by Frenkel and Turaev's summation formula (1.2), can be reduced to

$$
\frac{\left(a c, c / a, q^{\frac{3}{4}-\frac{n}{2}} z, q^{\frac{1}{4}-\frac{n}{2}} / z ; q, p\right)_{n}}{\left(c z, c / z, a q^{\frac{3}{4}-\frac{n}{2}}, q^{\frac{3}{4}-\frac{n}{2}} / a ; q, p\right)_{n}}
$$

Elementary manipulations can now be applied to transform this expression to the left-hand side of $(2.6)$.

\section{Expansions involving cubic theta functions}

The cubic theta function $\gamma(z, a ; p)$ with two independent variables $z$ and $a$ in addition to the nome $p$ was considered by S. Bhargava [3]. (For a thorough treatment of the theory of cubic theta functions in analogy to the theory of the classical Jacobi theta functions, see [22].) It is defined by

$$
\gamma(z, a ; p)=\sum_{k=-\infty}^{\infty} \sum_{l=-\infty}^{\infty} p^{k^{2}+k l+l^{2}} a^{k+l} z^{k-l} .
$$

This function, up to a normalization factor $\left(p^{2} ; p^{2}\right)_{\infty}^{2}$ (independent from $a$ and $z$ ), is almost equal to the following product of two modified Jacobi theta functions

$$
\left(p^{2} ; p^{2}\right)_{\infty}^{2} \theta\left(-p a z ; p^{2}\right) \theta\left(-p a / z ; p^{2}\right)=\sum_{k=-\infty}^{\infty} \sum_{l=-\infty}^{\infty} p^{k^{2}+l^{2}} a^{k+l} z^{k-l}
$$

which differs by the factor $p^{k l}$ to the summand of the double series in (3.1). Because of this additional factor $p^{k l}$, the cubic theta function does not factorize into a product of two modified Jacobi theta functions of such a simple form. In principle though, the cubic theta function could be factorized into two modified Jacobi theta functions, but their arguments would have nontrivial expansions in $a, z$, and $p$.

From $(3.1)$, by replacing $(k, l)$ by $(l, k)$, or $(k, l)$ by $(-l,-k)$, respectively, we immediately deduce the symmetries [3]

$$
\gamma(1 / z, a ; p)=\gamma(z, a ; p)
$$

and

$$
\gamma(z, 1 / a ; p)=\gamma(z, a ; p)
$$

Further, from (3.1), by replacing $(k, l)$ by $(k+\lambda+\mu, l+\lambda)$, it is easy to verify that for all integers $\lambda$ and $\mu$ the following functional equation holds [3]:

$$
\gamma(z, a ; p)=p^{3 \lambda^{2}+3 \lambda \mu+\mu^{2}} a^{2 \lambda+\mu} z^{\mu} \gamma\left(p^{\mu / 2} z, p^{3(2 \lambda+\mu) / 2} a ; p\right) .
$$


In particular, we have the quasi periodicities

$$
\gamma(p z, a ; p)=\frac{1}{p z^{2}} \gamma(z, a ; p)
$$

and

$$
\gamma\left(z, p^{3} a ; p\right)=\frac{1}{p^{3} a^{2}} \gamma(z, a ; p) .
$$

Further, by separating the terms in the expansion of $p$ according to whether the exponents of $p$ are divisible by 3 or not, one can show [3]

$$
\gamma(z, a ; p)=\gamma\left(\sqrt{a z^{3}}, \sqrt{a^{3} / z^{3}} ; p^{3}\right)+p a z^{-1} \gamma\left(\sqrt{a z^{3}}, p^{3} \sqrt{a^{3} / z^{3}} ; p^{3}\right),
$$

while separating the terms in the expansion of $z$ according to whether the exponents of $z$ are even or odd, one has [4]

$$
\gamma(z, a ; p)=\left(p^{6} ; p^{6}\right)_{\infty}\left(p^{2} ; p^{2}\right)_{\infty}\left[\theta\left(-p^{3} a ; p^{6}\right) \theta\left(-p z^{2} ; p^{2}\right)+p a z \theta\left(-p^{6} a^{2} ; p^{6}\right) \theta\left(-p^{2} z^{2} ; p^{2}\right)\right] .
$$

Cooper and Toh [6] proved the following addition formulae which will be useful in our computations.

Lemma 3.1 ([6, Corollary 4.5]). The following identities connecting modified Jacobi theta functions and cubic theta functions hold:

$$
\begin{gathered}
\gamma\left(z_{1}, \alpha ; p\right) \theta\left(z_{3} / z_{2}, z_{2} z_{3} ; p\right)-\gamma\left(z_{2}, \alpha ; p\right) \theta\left(z_{3} / z_{1}, z_{1} z_{3} ; p\right) \\
\quad=\frac{z_{3}}{z_{1}} \gamma\left(z_{3}, \alpha ; p\right) \theta\left(z_{1} / z_{2}, z_{1} z_{2} ; p\right)
\end{gathered}
$$

and

$$
\begin{gathered}
\gamma\left(z, \alpha_{1} ; p^{\frac{1}{3}}\right) \theta\left(\alpha_{3} / \alpha_{2}, \alpha_{2} \alpha_{3} ; p\right)-\gamma\left(z, \alpha_{2} ; p^{\frac{1}{3}}\right) \theta\left(\alpha_{3} / \alpha_{1}, \alpha_{1} \alpha_{3} ; p\right) \\
=\frac{\alpha_{3}}{\alpha_{1}} \gamma\left(z, \alpha_{3} ; p^{\frac{1}{3}}\right) \theta\left(\alpha_{1} / \alpha_{2}, \alpha_{1} \alpha_{2} ; p\right) .
\end{gathered}
$$

These two identities were proved in [6] by specializing a $(3 \times 3)$ determinant evaluation involving cubic theta functions. They can also be proved directly, expanding the cubic theta functions and modified Jacobi theta functions as infinite series, together with clever series rearrangement.

Now we introduce the first cubic theta analogue of the $q$-shifted factorial by

$$
\langle a z, a / z ; q, p\rangle_{n}:=\prod_{j=0}^{n-1} \gamma\left(z q^{\frac{1-n}{2}+j}, a q^{\frac{n-1}{2}} ; p\right) .
$$

From (3.3a) it is easy to see that the cubic shifted factorial satisfies

$$
\langle a p z, a / p z ; q, p\rangle_{n}=\frac{1}{p^{n} z^{2 n}}\langle a z, a / z ; q, p\rangle_{n} .
$$

Together with (3.2a), this implies that the quotient

$$
\frac{\langle a z, a / z ; q, p\rangle_{n}}{(c z, c / z ; q, p)_{n}}
$$

is in the space $W_{c}^{n}$. Hence we can apply Theorem 2.2 to it, by which we obtain the first cubic theta extension of Jackson's ${ }_{8} \phi_{7}$ summation (1.3). 
Corollary 3.2. We have the following summation

$$
\begin{aligned}
& (b c, c / b ; q, p)_{n} \frac{\langle a z, a / z ; q, p\rangle_{n}}{(c z, c / z ; q, p)_{n}}=\sum_{k=0}^{n} q^{n k}\left(\frac{c}{b}\right)^{k} \frac{\theta\left(b c q^{2 k-1} ; p\right)}{\theta\left(b c q^{-1} ; p\right)} \frac{\left(q^{-n}, b c q^{-1}, b z, b / z ; q, p\right)_{k}}{\left(q, b c q^{n}, c z, c / z ; q, p\right)_{k}} \\
& \quad \times\left\langle a c q^{n-1}, a q^{1-k} / c ; q, p\right\rangle_{k} \frac{\left\langle a b q^{k}, a q^{-k} / b ; q, p\right\rangle_{n}}{\left\langle a b q^{n}, a q^{-k} / b ; q, p\right\rangle_{k}} .
\end{aligned}
$$

Proof. By using (3.4a) in Lemma 3.1, we can prove by induction that

$$
\begin{aligned}
\mathcal{D}_{c, q, p}^{(k)} & \left(\frac{\langle a z, a / z ; q, p\rangle_{n}}{(c z, c / z ; q, p)_{n}}\right) \\
= & (2 c)^{k} q^{\frac{3}{4} k(k-1)} \frac{\left(q^{n} ; q^{-1}, p\right)_{k}}{\theta(q ; p)^{k}} \prod_{j=0}^{k-1} \gamma\left(c q^{\frac{n-1}{2}+j}, a q^{\frac{n-1}{2}} ; p\right) \frac{\left\langle a q^{\frac{k}{2}} z, a q^{\frac{k}{2}} / z ; q, p\right\rangle_{n-k}}{\left(c q^{\frac{3}{2} k} z, c q^{\frac{3}{2} k} / z ; q, p\right)_{n-k}} \\
= & (2 c)^{k} q^{\frac{3}{4} k(k-1)} \frac{\left(q^{n} ; q^{-1}, p\right)_{k}}{\theta(q ; p)^{k}}\left\langle a c q^{n-1}, a q^{1-k} / c ; q, p\right\rangle_{k} \frac{\left\langle a q^{\frac{k}{2}} z, a q^{\frac{k}{2}} / z ; q, p\right\rangle_{n-k}}{\left(c q^{\frac{3}{2} k} z, c q^{\frac{3}{2} k} / z ; q, p\right)_{n-k}} .
\end{aligned}
$$

Then the corollary follows from Theorem 2.2 while expanding in the basis

$$
f(z)=\frac{(b z, b / z ; q, p)_{n}}{(c z, c / z ; q, p)_{n}} .
$$

To recover Jackson's ${ }_{8} \phi_{7}$ summation from Corollary 3.2, substitute

$$
a \mapsto \frac{-a}{p\left(1+a^{2} q^{n-1}\right)}
$$

in (3.5), multiply both sides of the identity by $\left(1+a^{2} q^{n-1}\right)^{n}$ and let $p \rightarrow 0$. When $p \rightarrow 0$, the usual theta shifted factorials clearly reduce to the $q$-shifted factorials. That is, the quotient on the left-hand side reduces to

$$
\lim _{p \rightarrow 0} \frac{(b c, c / b ; q, p)_{n}}{(c z, c / z ; q, p)_{n}}=\frac{(b c, c / b ; q)_{n}}{(c z, c / z ; q)_{n}} .
$$

What happens with the cubic theta shifted factorial? We have

$$
\begin{aligned}
& \lim _{p \rightarrow 0}\left(1+a^{2} q^{n-1}\right)^{n}\left\langle\frac{-a z}{p\left(1+a^{2} q^{n-1}\right)}, \frac{-a}{p\left(1+a^{2} q^{n-1}\right) z} ; p\right\rangle_{n} \\
& =\left(1+a^{2} q^{n-1}\right)^{n} \lim _{p \rightarrow 0} \prod_{j=0}^{n-1} \gamma\left(z q^{\frac{1-n}{2}+j}, \frac{-a q^{\frac{n-1}{2}}}{p\left(1+a^{2} q^{n-1}\right)} ; p\right) \\
& =\left(1+a^{2} q^{n-1}\right)^{n} \prod_{j=0}^{n-1} \lim _{p \rightarrow 0} \sum_{k=-\infty}^{\infty} \sum_{l=-\infty}^{\infty}(-1)^{k+l} p^{k^{2}+k l+l^{2}-k-l}\left(\frac{a q^{\frac{n-1}{2}}}{1+a^{2} q^{n-1}}\right)^{k+l}\left(z q^{\frac{1-n}{2}+j}\right)^{k-l} .
\end{aligned}
$$

Now it is easy to see that for $p \rightarrow 0$ only three terms in the various double infinite series survive. These three terms correspond to the cases $(k, l)=(0,0),(1,0),(0,1)$. The last expression thus reduces to

$$
\begin{aligned}
(1+ & \left.a^{2} q^{n-1}\right)^{n} \prod_{j=0}^{n-1}\left(1-\frac{a q^{\frac{n-1}{2}}}{1+a^{2} q^{n-1}}\left(z q^{\frac{1-n}{2}+j}+z^{-1} q^{\frac{n-1}{2}-j}\right)\right) \\
& =\prod_{j=0}^{n-1}\left(1+a^{2} q^{n-1}-a q^{\frac{n-1}{2}}\left(z q^{\frac{1-n}{2}+j}+z^{-1} q^{\frac{n-1}{2}-j}\right)\right) \\
& =\prod_{j=0}^{n-1}\left(1-a z q^{j}\right)\left(1-a q^{n-1-j} / z\right)=(a z, a / z ; q)_{n} .
\end{aligned}
$$

We take similar limits on the right-hand side of (3.5). 
Our next result involves elliptic interpolation of cubic theta shifted factorials.

Corollary 3.3. We have the following Karlsson-Minton type identity involving cubic theta functions

$$
\begin{aligned}
& \frac{\left(a^{2} q, q ; q, p\right)_{n}}{(a q z, a q / z ; q, p)_{n}}\langle b z, b / z ; q, p\rangle_{n} \\
& \quad=\sum_{k=0}^{n} q^{k(n+1)} \frac{\theta\left(a^{2} q^{2 k} ; p\right)}{\theta\left(a^{2} ; p\right)} \frac{\left(q^{-n}, a^{2}, a z, a / z ; q, p\right)_{k}}{\left(q, a^{2} q^{n+1}, a q z, a q / z ; q, p\right)_{k}}\left\langle a b q^{k}, b q^{-k} / a ; q, p\right\rangle_{n} .
\end{aligned}
$$

Proof. We apply Theorem 2.6 to

$$
f(z)=\frac{\langle b z, b / z ; q, p\rangle_{n}}{(c z, c / z ; q, p)_{n}}
$$

More generally, we have the following Karlsson-Minton type identity involving cubic theta functions.

Corollary 3.4. We have

$$
\begin{aligned}
& \frac{\left(a^{2} q, q ; q, p\right)_{n}}{(a q z, a q / z ; q, p)_{n}} \prod_{i=1}^{s} \theta\left(b_{i} z, b_{i} / z ; p\right) \prod_{j=1}^{n-s} \gamma\left(z, d_{j} ; p\right)=\sum_{k=0}^{n} q^{k(n+1)} \frac{\theta\left(a^{2} q^{2 k} ; p\right)}{\theta\left(a^{2} ; p\right)} \\
& \times \frac{\left(q^{-n}, a^{2}, a z, a / z ; q, p\right)_{k}}{\left(q, a^{2} q^{n+1}, a q z, a q / z ; q, p\right)_{k}} \prod_{i=1}^{s} \theta\left(a b_{i} q^{k}, b_{i} q^{-k} / a ; p\right) \prod_{j=1}^{n-s} \gamma\left(a q^{k}, d_{j} ; p\right) .
\end{aligned}
$$

Proof. We apply Theorem 2.6 to

$$
f(z)=\frac{\prod_{i=1}^{s} \theta\left(b_{i} z, b_{i} / z ; p\right) \prod_{j=1}^{n-s} \gamma\left(z, d_{j} ; p\right)}{(c z, c / z ; q, p)_{n}} .
$$

Our next result concerns a cubic theta extension of Gessel and Stanton's quadratic summation [10, equation (1.4)].

Corollary 3.5. We have the following summation

$$
\begin{aligned}
& \frac{\langle a z, a / z ; q, p\rangle_{n}}{(c z, c / z ; q, p)_{n}}\left(c q^{-\frac{1}{4}}, c q^{\frac{1}{4}} ; q, p\right)_{n} \\
& \quad=\sum_{k=0}^{n} c^{k} q^{\frac{k}{4}(k-2)+n k} \frac{\theta\left(c q^{\frac{3}{2} k-\frac{3}{4}} ; p\right)}{\theta\left(c q^{\frac{k}{2}-\frac{3}{4}} ; p\right)} \frac{\left(q^{-n} ; q, p\right)_{k}}{(q ; q, p)_{k}} \frac{\left(c q^{-\frac{1}{4}} ; q^{\frac{1}{2}}, p\right)_{k}}{\left(c q^{n-\frac{1}{4}} ; q^{\frac{1}{2}}, p\right)_{k}} \frac{\left(q^{\frac{1}{4}} z, q^{\frac{1}{4}} / z ; q^{\frac{1}{2}}, p\right)_{k}}{(c z, c / z ; q, p)_{k}} \\
& \quad \times\left\langle a c q^{n-1}, a q^{1-k} / c ; q, p\right\rangle_{k}\left\langle a q^{\frac{k}{2}+\frac{1}{4}}, a q^{\frac{k}{2}-\frac{1}{4}} ; q, p\right\rangle_{n-k} .
\end{aligned}
$$

Proof. We apply Theorem 2.15 to

$$
f(z)=\frac{\langle a z, a / z ; q, p\rangle_{n}}{(c z, c / z ; q, p)_{n}}
$$

Similarly to the way we recovered Jackson's ${ }_{8} \phi_{7}$ summation from Corollary 3.2 , Gessel and Stanton's quadratic summation can be readily obtained by substituting $a \mapsto-p^{-1} a /\left(1+a^{2} q^{n-1}\right)$ in (3.6), multiplying both sides by $\left(1+a^{2} q^{n-1}\right)^{n}$ and taking the limit $p \rightarrow 0$. 
Next, we define the second cubic theta shifted factorial, with base $p^{1 / 3}$ :

$$
\left\langle\left\langle a z, a / z ; q, p^{\frac{1}{3}}\right\rangle\right\rangle_{n}:=\prod_{j=0}^{n-1} \gamma\left(a q^{\frac{n-1}{2}}, z q^{\frac{1-n}{2}+j} ; p^{\frac{1}{3}}\right) .
$$

Recalling equations (3.3a) and (3.3b) (which we reformulate after interchanging $a$ and $z$ ),

$$
\gamma(a, z ; p)=\gamma(a, 1 / z ; p), \quad \gamma(a, z ; p)=p^{3} z^{2} \gamma\left(a, p^{3} z ; p\right),
$$

we see that

$$
\left\langle\left\langle a p z, a / p z ; q, p^{\frac{1}{3}}\right\rangle\right\rangle_{n}=\frac{1}{p^{n} z^{2 n}}\left\langle\left\langle a z, a / z ; q, p^{\frac{1}{3}}\right\rangle\right\rangle_{n} .
$$

This implies that the quotient

$$
\frac{\left\langle\left\langle a z, a / z ; q, p^{\frac{1}{3}}\right\rangle\right\rangle_{n}}{(c z, c / z ; q, p)_{n}}
$$

is also in the space $W_{c}^{n}$. Thus, Theorem 2.2 can be applied to it, by which we obtain the second cubic theta extension of Jackson's ${ }_{8} \phi_{7}$ summation (1.3).

Corollary 3.6. We have the following summation

$$
\begin{gathered}
\frac{\left\langle\left\langle b z, b / z ; q, p^{\frac{1}{3}}\right\rangle\right\rangle_{n}}{(c z, c / z ; q, p)_{n}}(a c, c / a ; q, p)_{n}=\sum_{k=0}^{n} q^{n k}\left(\frac{c}{a}\right)^{k} \frac{\theta\left(a c q^{2 k-1} ; p\right)}{\theta\left(a c q^{-1} ; p\right)} \frac{\left(q^{-n}, a c q^{-1}, a z, a / z ; q, p\right)_{k}}{\left(q, a c q^{n}, c z, c / z ; q, p\right)_{k}} \\
\times\left\langle\left\langle b c q^{n-1}, b q^{1-k} / c ; q, p^{\frac{1}{3}}\right\rangle\right\rangle_{k}\left\langle\left\langle a b q^{k}, b / a ; q, p^{\frac{1}{3}}\right\rangle\right\rangle_{n-k} .
\end{gathered}
$$

Proof. Note that by using (3.4b) in Lemma 3.1, we can show by induction that

$$
\begin{aligned}
\mathcal{D}_{c, q, p}^{(k)} & \left(\frac{\left\langle\left\langle b z, b / z ; q, p^{\frac{1}{3}}\right\rangle\right\rangle_{n}}{(c z, c / z ; q, p)_{n}}\right) \\
= & (2 c)^{k} q^{\frac{3}{4} k(k-1)} \frac{\left(q^{n} ; q^{-1}, p\right)_{k}}{\theta(q ; p)^{k}} \prod_{j=0}^{k-1} \gamma\left(b q^{\frac{n-1}{2}}, c q^{\frac{n-1}{2}+j} ; p^{\frac{1}{3}}\right) \frac{\left\langle\left\langle b q^{\frac{k}{2}} z, b q^{\frac{k}{2}} / z ; q, p^{\frac{1}{3}}\right\rangle\right\rangle_{n-k}}{\left(c q^{\frac{3}{2}} z, c q^{\frac{3}{2} k} / z ; q, p\right)_{n-k}} \\
= & (2 c)^{k} q^{\frac{3}{4} k(k-1)} \frac{\left(q^{n} ; q^{-1}, p\right)_{k}}{\theta(q ; p)^{k}}\left\langle\left\langle b c q^{n-1}, b q^{1-k} / c ; q, p^{\frac{1}{3}}\right\rangle\right\rangle_{k} \frac{\left\langle\left\langle b q^{\frac{k}{2}} z, b q^{\frac{k}{2}} / z ; q, p^{\frac{1}{3}}\right\rangle\right\rangle_{n-k}}{\left(c q^{\frac{3}{2} k} z, c q^{\frac{3}{2} k} / z ; q, p\right)_{n-k}} .
\end{aligned}
$$

Using this, we apply Theorem 2.2 to

$$
f(z)=\frac{\left\langle\left\langle b z, b / z ; q, p^{\frac{1}{3}}\right\rangle\right\rangle_{n}}{(c z, c / z ; q, p)_{n}} .
$$

To recover Jackson's ${ }_{8} \phi_{7}$ summation from Corollary 3.6, substitute

$$
b \mapsto \frac{-b}{p^{\frac{1}{3}}\left(1+b^{2} q^{n-1}\right)}
$$

in (3.7), multiply both sides of the identity by $\left(1+b^{2} q^{n-1}\right)^{n}$ and let $p \rightarrow 0$. When $p \rightarrow 0$, the usual theta shifted factorials reduce to the $q$-shifted factorials and the cubic theta shifted factorial on the left-hand side of (3.7) becomes

$$
\lim _{p \rightarrow 0}\left(1+b^{2} q^{n-1}\right)^{n}\left\langle\left\langle\frac{-b z}{p^{\frac{1}{3}}\left(1+b^{2} q^{n-1}\right)}, \frac{-b}{p^{\frac{1}{3}}\left(1+b^{2} q^{n-1}\right) z} ; p^{\frac{1}{3}}\right\rangle\right\rangle_{n}
$$




$$
\begin{aligned}
= & \left(1+b^{2} q^{n-1}\right)^{n} \lim _{p \rightarrow 0} \prod_{j=0}^{n-1} \gamma\left(\frac{-b q^{\frac{n-1}{2}}}{p^{\frac{1}{3}}\left(1+b^{2} q^{n-1}\right)}, z q^{\frac{1-n}{2}+j} ; p^{\frac{1}{3}}\right) \\
= & \left(1+b^{2} q^{n-1}\right)^{n} \prod_{j=0}^{n-1} \lim _{p \rightarrow 0} \sum_{k=-\infty}^{\infty} \sum_{l=-\infty}^{\infty}(-1)^{k-l} p^{\frac{1}{3}\left(k^{2}+k l+l^{2}-k+l\right)} \\
& \times\left(z q^{\frac{1-n}{2}+j}\right)^{k+l}\left(\frac{b q^{\frac{n-1}{2}}}{1+b^{2} q^{n-1}}\right)^{k-l} .
\end{aligned}
$$

Now it is easy to see that for $p \rightarrow 0$ only three terms in the various double infinite series survive. These correspond to the cases $(k, l)=(0,0),(1,0),(0,-1)$. The last expression thus reduces to

$$
\begin{aligned}
(1+ & \left.b^{2} q^{n-1}\right)^{n} \prod_{j=0}^{n-1}\left(1-\frac{b q^{\frac{n-1}{2}}}{1+b^{2} q^{n-1}}\left(z q^{\frac{1-n}{2}+j}+z^{-1} q^{\frac{n-1}{2}-j}\right)\right) \\
& =\prod_{j=0}^{n-1}\left(1+b^{2} q^{n-1}-b q^{\frac{n-1}{2}}\left(z q^{\frac{1-n}{2}+j}+z^{-1} q^{\frac{n-1}{2}-j}\right)\right) \\
& =\prod_{j=0}^{n-1}\left(1-b z q^{j}\right)\left(1-b q^{n-1-j} / z\right)=(b z, b / z ; q)_{n} .
\end{aligned}
$$

We take similar limits on the right-hand side of equation (3.7).

Our final result concerns another cubic theta extension of Gessel and Stanton's quadratic summation [10, equation (1.4)].

Corollary 3.7. We have the following summation

$$
\begin{aligned}
& \frac{\left\langle\left\langle a z, a / z ; q, p^{\frac{1}{3}}\right\rangle\right\rangle_{n}}{(c z, c / z ; q, p)_{n}}\left(c q^{-\frac{1}{4}}, c q^{\frac{1}{4}} ; q, p\right)_{n} \\
& =\sum_{k=0}^{n} c^{k} q^{\frac{k}{4}(k-2)+n k} \frac{\theta\left(c q^{\frac{3}{2} k-\frac{3}{4}} ; p\right)}{\theta\left(c q^{\frac{k}{2}-\frac{3}{4}} ; p\right)} \frac{\left(q^{-n} ; q, p\right)_{k}}{(q ; q, p)_{k}} \frac{\left(c q^{-\frac{1}{4}} ; q^{\frac{1}{2}}, p\right)_{k}}{\left(c q^{n-\frac{1}{4}} ; q^{\frac{1}{2}}, p\right)_{k}} \frac{\left(q^{\frac{1}{4}} z, q^{\frac{1}{4}} / z ; q^{\frac{1}{2}}, p\right)_{k}}{(c z, c / z ; q, p)_{k}} \\
& \quad \times\left\langle\left\langle a c q^{n-1}, a q^{1-k} / c ; q, p^{\frac{1}{3}}\right\rangle\right\rangle_{k}\left\langle\left\langle a q^{\frac{k}{2}+\frac{1}{4}}, a q^{\frac{k}{2}-\frac{1}{4}} ; q, p^{\frac{1}{3}}\right\rangle\right\rangle_{n-k} .
\end{aligned}
$$

Proof. We apply Theorem 2.15 to

$$
f(z)=\frac{\left\langle\left\langle a z, a / z ; q, p^{\frac{1}{3}}\right\rangle\right\rangle_{n}}{(c z, c / z ; q, p)_{n}} .
$$

\section{Acknowledgements}

The work in this paper has been supported by FWF Austrian Science Fund grant F50-08 within the SFB "Algorithmic and enumerative combinatorics".

\section{References}

[1] Askey R., Wilson J., Some basic hypergeometric orthogonal polynomials that generalize Jacobi polynomials, Mem. Amer. Math. Soc. 54 (1985), no. 319, iv+55 pages.

[2] Baxter R.J., Eight-vertex model in lattice statistics and one-dimensional anisotropic Heisenberg chain. II. Equivalence to a generalized ice-type model, Ann. Physics 76 (1973), 25-47.

[3] Bhargava S., Unification of the cubic analogues of the Jacobian theta-function, J. Math. Anal. Appl. 193 (1995), 543-558. 
[4] Bhargava S., Fathima S.N., Laurent coefficients for cubic theta functions, South East Asian J. Math. Math. Sci. 1 (2003), 27-31.

[5] Cooper S., The Askey-Wilson operator and the ${ }_{6} \Phi_{5}$ summation formula, South East Asian J. Math. Math. Sci. 1 (2002), 71-82.

[6] Cooper S., Toh P.C., Determinant identities for theta functions, J. Math. Anal. Appl. 347 (2008), 1-7.

[7] Date E., Jimbo M., Kuniba A., Miwa T., Okado M., Exactly solvable SOS models: local height probabilities and theta function identities, Nuclear Phys. B 290 (1987), 231-273.

[8] Frenkel I.B., Turaev V.G., Elliptic solutions of the Yang-Baxter equation and modular hypergeometric functions, in The Arnold-Gelfand Mathematical Seminars, Editors V.I. Arnold, I.M. Gelfand, V.S. Retakh, M. Smirnov, Birkhäuser Boston, Boston, MA, 1997, 171-204.

[9] Gasper G., Rahman M., Basic hypergeometric series, Encyclopedia of Mathematics and its Applications, Vol. 96, 2nd ed., Cambridge University Press, Cambridge, 2004.

[10] Gessel I., Stanton D., Applications of $q$-Lagrange inversion to basic hypergeometric series, Trans. Amer. Math. Soc. 277 (1983), 173-201.

[11] Ismail M.E.H., The Askey-Wilson operator and summation theorems, in Mathematical Analysis, Wavelets, and Signal Processing (Cairo, 1994), Contemp. Math., Vol. 190, Amer. Math. Soc., Providence, RI, 1995, $171-178$.

[12] Ismail M.E.H., Rains E.M., Stanton D., Orthogonality of very-well-poised series, Int. Math. Res. Not., to appear.

[13] Ismail M.E.H., Stanton D., Applications of q-Taylor theorems, J. Comput. Appl. Math. 153 (2003), 259-272.

[14] Ismail M.E.H., Stanton D., Some combinatorial and analytical identities, Ann. Comb. 16 (2012), 755-771.

[15] Marco J.M., Parcet J., A new approach to the theory of classical hypergeometric polynomials, Trans. Amer. Math. Soc. 358 (2006), 183-214, math.CO/0312247.

[16] Rains E.M., $B C_{n}$-symmetric Abelian functions, Duke Math. J. 135 (2006), 99-180, math.CO/0402113.

[17] Rains E.M., Transformations of elliptic hypergeometric integrals, Ann. of Math. 171 (2010), 169-243, math.QA/0309252.

[18] Rosengren H., Sklyanin invariant integration, Int. Math. Res. Not. 2004 (2004), 3207-3232, math.QA/0405072.

[19] Rosengren H., An elementary approach to $6 j$-symbols (classical, quantum, rational, trigonometric, and elliptic), Ramanujan J. 13 (2007), 131-166, math.CA/0312310.

[20] Rosengren H., Schlosser M., Elliptic determinant evaluations and the Macdonald identities for affine root systems, Compos. Math. 142 (2006), 937-961, math.CA/0505213.

[21] Schlosser M.J., A Taylor expansion theorem for an elliptic extension of the Askey-Wilson operator, in Special Functions and Orthogonal Polynomials, Contemp. Math., Vol. 471, Amer. Math. Soc., Providence, RI, 2008, 175-186, arXiv:0803.2329.

[22] Schultz D., Cubic theta functions, Adv. Math. 248 (2013), 618-697.

[23] Spiridonov V.P., Theta hypergeometric series, in Asymptotic Combinatorics with Application to Mathematical Physics (St. Petersburg, 2001), NATO Sci. Ser. II Math. Phys. Chem., Vol. 77, Editors V.A. Malyshev, A.M. Vershik, Kluwer Acad. Publ., Dordrecht, 2002, 307-327, math.CA/0303204.

[24] Warnaar S.O., Summation and transformation formulas for elliptic hypergeometric series, Constr. Approx. 18 (2002), 479-502, math.QA/0001006.

[25] Weber H., Elliptische Functionen und Algebraische Zahlen, Vieweg u. Sohn, Braunschweig, 1891.

[26] Whittaker E.T., Watson G.N., A course of modern analysis, Cambridge Mathematical Library, Cambridge University Press, Cambridge, 1996. 